\title{
Molecular

\section{Large-scale network models of IL-1 and IL-6 signalling and their hepatocellular specification $\dagger$}

\author{
Anke Ryll, ${ }^{a}$ Regina Samaga, ${ }^{a}$ Fred Schaper, ${ }^{b}$ Leonidas G. Alexopoulos ${ }^{c}$ and \\ Steffen Klamt*a
}

Received 24th June 2011, Accepted 6th September 2011

DOI: $10.1039 / \mathrm{c} 1 \mathrm{mb05261f}$

The pro-inflammatory cytokines interleukin 1 (IL-1) and 6 (IL-6) are crucially involved in the regulation of a multitude of physiological processes, in particular coordinating the immune response upon bacterial infection and tissue injury. Both interleukins induce complex signalling cascades and trigger the production of mitogenic, pro-proliferative, anti-apoptotic, chemotactic, and pro-angiogenic factors thereby affecting the delicate balance between regeneration $v s$. invasive growth, tumourigenesis and metastasis. Moreover, several links to insulin resistance have been found within their associated signalling networks. Focusing on this from a systems biology perspective, we introduce comprehensive large-scale network models of IL-1 and IL-6 signalling which are based on a logical modelling approach and reflect the current biological knowledge. Theoretical network analysis enabled us to uncover general topological features and to make testable predictions on the stimulus-response behaviour of the networks. In this context, non-intuitive network-wide species dependencies as well as structures of regulatory feedback and feed-forward mechanisms could be characterised. By integrating high-throughput phosphoproteomic data from primary human hepatocytes we optimised the model structures to obtain models with high prediction accuracy for hepatocytes. Our model-based data analysis, for instance, suggested model modifications regarding (i) Akt contribution to IL-1-stimulated p38 MAPK activation and (ii) insignificant p38 MAPK activation in response to IL-6. In light of the presented results and in conjunction with the detailed model documentations, both models hold great potential for theoretical studies and practical applications.

\section{Introduction}

The two pleiotropic factors of inflammatory response, interleukin 1 (IL-1) and 6 (IL-6) are induced and expressed by a wide range of cell types, including monocytes, endothelial cells, and fibroblasts in response to, for example, endotoxic stimulus exposure or stimulation with initiators of sequential inflammatory cytokine release such as tumour necrosis factor (TNF) and interferon (IFN) isoforms. ${ }^{1-5}$ Both interleukins control hepatic acute-phase protein (APP) secretion ${ }^{6-10}$ during innate immune response upon bacterial infection and tissue injury, facilitating regeneration and wound healing. The IL-1-induced release of potent immune attractors and/or chemokines (e.g. CCL2 (MCP1), IL-8) $)^{11,12}$ triggers transmigration of

${ }^{a}$ Max Planck Institute for Dynamics of Complex Technical Systems, Sandtorstrasse 1, D-39106 Magdeburg, Germany.

E-mail:klamt@mpi-magdeburg.mpg.de

${ }^{b}$ Otto-von-Guericke University Magdeburg, Institute of Biology, Leipziger Strasse 44, D-39120 Magdeburg, Germany

${ }^{c}$ National Technical University of Athens, Department of Mechanical Engineering, Athens, 15780, Greece

$\dagger$ Electronic supplementary information (ESI) available. See DOI: $10.1039 / \mathrm{clmb} 05261 \mathrm{f}$ immunocompetent cells (e.g. activated macrophages, granulocytes) to sites of inflammation. Moreover, IL-1 and IL-6 may effect the delicate balance between regeneration vs. invasive growth, tumourigenesis, and metastasis ${ }^{13,14}$ in conjunction with hepatitis, ischemia, and cirrhosis ${ }^{15,16}$ by up-regulating the production of mitogenic, pro-proliferative, anti-apoptotic, chemotactic, and pro-angiogenic growth factors like HGF. ${ }^{17-19}$ Apart from that, JNK and IKK $\beta$ activation by IL- $1^{20-22}$ as well as SOCS expression $^{23,24}$ or SHP2 phosphatase $^{25}$ and ERK kinase ${ }^{26}$ activities induced by IL-6 stimulation link these ligands to insulin desensitisation and subsequent insulin resistance.

In this work, we introduce large-scale Boolean network models of IL-1 and IL-6 signalling manually compiled from literature and database knowledge. Comprehensive pathway diagrams such as the IL-1 signalling map by Kracht et al. ${ }^{27,28}$ served as starting points and valuable information sources for model construction. The fact that both interleukins share common signalling cascades and effectors (e.g. PI3K/Akt, MEK1/ERK, SOCS1/3) prompted us to study their associated signal transduction networks in parallel. A qualitative (parameter-free) modelling approach previously applied to T cell receptor ${ }^{29}$ and EGFR/ ErbB signalling ${ }^{30}$ was adopted, providing the mathematical 
framework for the two "master models" represented here. Subsequent network analysis aimed at the following three major objectives: (i) examination of general IL-1/IL-6 network properties, (ii) analysis of crosstalk strategies of both cytokines as well as their influences on HGF- and insulin-induced signalling pathways, and (iii) comparison of qualitative model predictions with phosphoproteomic data from primary human hepatocytes to delineate hepatocellular specifics within IL-1 and IL-6 signal propagation.

Each model comprises more than 70 nodes (e.g. scaffold proteins, kinases, transcription factors) and 80 interactions. The high number of species and reactions along with insufficient information on reaction kinetics and protein concentrations impedes quantitative and time-resolved descriptions of large-scale signalling networks by ordinary differential equations (ODEs). Consequently, our models focus on the wiring diagrams of as well as Boolean relationships in IL-1 and IL-6 signalling. This representation not only suits to uncover major players and interactions but also to reveal network-wide interdependencies. Furthermore, it serves as an "executable pathway map" to study stimulus-response (input-output) behaviours or to compute minimal intervention sets (MIS) ${ }^{31}$ that enforce a predefined cellular response.

The literature-derived network models presented here capture the current state of biological knowledge concerning IL-1 and IL-6 signalling pathways to an extent that has not been described before, particularly regarding IL-6. We validated the network structures by means of high-throughput experimental data from primary human hepatocytes exposed to specific ligand/intracellular kinase inhibitor combinations. ${ }^{32}$ Detected discrepancies between data and "master model" predictions were used for topological optimisation to obtain specific models reflecting the hepatocellular phenotype.

\section{Results}

As a first step, we reconstructed two Boolean "master models" for IL-1 (model M1; Fig. 1) and IL-6 (model M2; Fig. 2) by merging biological knowledge from various cell types as provided by databases and scientific literature (see supplementary model documentations, ESI $\dagger$ ). Both models are represented as logical interaction hypergraphs (LIHs, cf. "Methods/ Model set-up") allowing efficient visualisation and storage of logical relationships. ${ }^{31}$ Excluding dummy variables and reservoirs ( $c f$. "Methods"), M1 (M2) contains 97 (75) nodes or species and 113 (84) interactions. 17 (15) nodes are inputs to the model not regulated within the scope of the network (e.g. ligands, receptor subunits, and regulatory phosphatases) and therefore predefined by default values (see Tables S3.1 and S4.1, ESI $\dagger$ ) during simulations. 12 (12) nodes represent outputs, mainly depicting APPs, cytokines, and transcription factors. Each interaction was additionally characterised by a confidence level given in Tables S3.2 and S4.2 (ESI $\dagger$ ) and colour-coded in Fig. 1 and 2, denoting whether an effect has already been shown (with respect to cited sources) for hepatocytes or for other cell types stimulated with IL-1 or IL-6. 11 (8) interactions were integrated by means of incomplete truth table (ITT) operators as physiological evidence for a clear distinction between AND or OR connections could not be derived from the literature. Furthermore, we classified 21 (16) interactions as secondary or "post-initial" events (e.g. interactions closing feedback loops), therefore setting their respective relevance parameter to $\tau=2$ (see also "Methods/Model set-up"). The logical analysis represented here principally focused on initial signal propagation scenarios and how they result in transcriptional activity and/or gene expression. " $\tau=2$ " interactions indicate effects that do not influence the initial systemic behaviour but may contribute to system dynamics at later time points. Nevertheless, we kept those events in the model, e.g. for studying the feedback structure of the networks, whereas excluded them when doing logical simulations.

Importantly, as described by Klamt et al., ${ }^{30,31}$ a Boolean model can be analysed either directly by using its logical description or with respect to its underlying interaction graph (IG), comprising no explicit logical statements and edges, hence capturing nothing but influences. IGs are thus simpler representations of signalling topologies, however, they facilitate studies on important topological properties such as feedback loops or interdependencies. Deriving the associated IG from a logical model is trivial as long as the latter is given in LIH representation ( $c f$. "Methods"). Accordingly, both IG and LIH representation are used for model and data analysis described below.

Model set-up and visualisation was realised with ProMoT, ${ }^{33-35}$ whereas qualitative analyses were performed using CellNetAnalyzer (CNA) $)^{36,37}$ (see also "Methods/Model set-up").

\section{Topological features revealed by interaction graph analysis}

In order to analyse intrinsic topological properties (e.g. feedback loops and global species dependencies) independently of any Boolean description, we first studied the directed interaction graphs ${ }^{31}$ uniquely underlying the logical models M1 (IL-1) and M2 (IL-6) henceforth referred to as IG1 and IG2 respectively (see also "Methods"). Generally, IGs solely cover pairwise positive or negative effects between species within a network, irrespectively of the deterministic logic functions chosen. Feedback loops (FLs) essentially affect system dynamics, on the one hand amplifying input signals and inducing multistationarity (both being relevant for cellular differentiation and decision processes) if positive, whereas ensuring cellular homoeostasis or re-sensitisation and possibly being accompanied by oscillatory behaviour if negative. ${ }^{38-41}$ The interaction graph associated to IL-1 (IL-6) signalling comprises 16515 (350) feedback loops, thereof 49.9\% (39.1\%) negative. Fig. S1 (S2; see ESI $\dagger$ ) illustrates the individual FL participation of species involved in IL-1 (IL-6) signalling, revealing, for instance, the Y-phosphorylated IL-6 receptor complex, membrane-bound and phosphorylated Gabl adaptor proteins, and phosphatase SHP2 as "IL-6 feedback protagonists". With respect to IL-1 signalling, more than $80 \%$ of detected FLs require the involvement of mitogen-activated kinases TAK1, MEKK3, and p38, phosphatase MKP1, and IL-1 receptor antagonists. Negative feedback loops within signalling networks are typically composed of a forward path and a feedback (either a single edge or a path). Referring to our models and as delineated subsequently, the former is in all cases positive implying the latter to be negative. The most important 


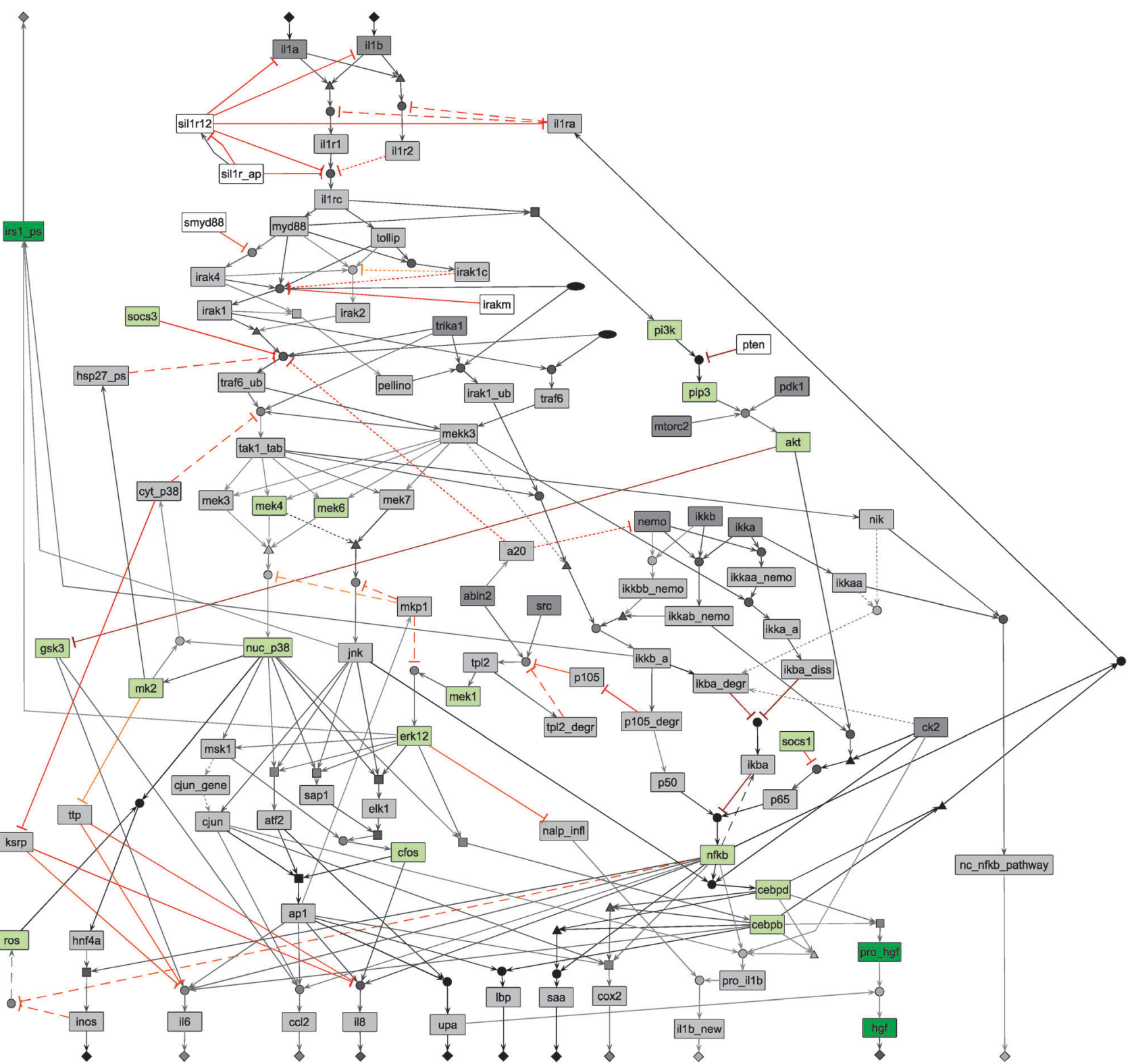

Fig. 1 Logical (master) model of IL-1 receptor signalling visualised in ProMoT. Signalling species are symbolised by rectangles and coloured according to functional involvement: white (dark grey): inhibitory (positive regulatory) inputs/side effectors with default value 0 (1); grey: species/ outputs regulated by up-stream effectors; light green: species acting in both networks (IL-1 and IL-6) revealing systemic crosstalks; dark green: species depicting links to insulin and HGF signalling. Reservoirs are described by black ellipses. Black arrows (red blunt-ended lines) indicate activations (inhibitions) with colour shades (grey-black $v$ s. orange-dark red) pointing to confidence levels $(0.4-1.0)$. Secondary events $(\tau=2)$ are displayed by dashed and dotted lines, on the one hand closing feedback loops (dashed), on the other hand seeming of minor initial relevance (dotted). Diamonds tag ligands and model outputs, whereas circles (squares) indicate logical AND (ITT) operators. Triangles represent OR terms contributing to complex AND connections. Again, symbol colours of aforementioned operators refer to confidence levels (see above). Dummy species are hidden for reasons of clarity. For detailed descriptions see "Methods" and supplemental model documentations, ESI. $\dagger$

negative feedbacks are: (i) activation of inhibitory phosphatases (IL-1: MKP1 vs. IL-6: SHP2), (ii) activation of kinases (IL-1: p38/MK2) S/T-phosphorylating and thereby negatively regulating essential scaffold proteins (IL-1: Hsp27, TAB) and receptor subunits (IL-6: gp130), (iii) up-regulation of inhibitory proteins (IL-1: $\mathrm{I} \kappa \mathrm{B} \alpha$ vs. IL-6: SOCS1/3) and receptor antagonists (IL-1: IL-1Ra), (iv) suppression of transcriptional activities (IL-1: HNF4 $\alpha$ ), due to elicitor (IL-1: ROS) "neutralisation" by up-regulated corresponding gene products (IL-1: iNOS), and (v) species degradation (IL-1: TPL2), inactivating downstream effectors (IL-1: ERK1/2). Most of the detected positive feedback loops consist of negative forward paths and subsequent negative feedbacks. Biologically speaking, activated phosphatases (IL-1: MKP1 vs. IL-6: SHP2) may not just counteract intracellular inhibitory kinase activities (e.g. inhibitory p38 activity; $c f$. (ii)) but also the inhibitory effect of SOCS1/3 and IL-1Ra, hence positively contributing to receptor function (Fig. 3A and B).

With respect to IL-6 signalling, PI3K- as well as ERKdependent Gab1 membrane translocation and phosphorylation, 


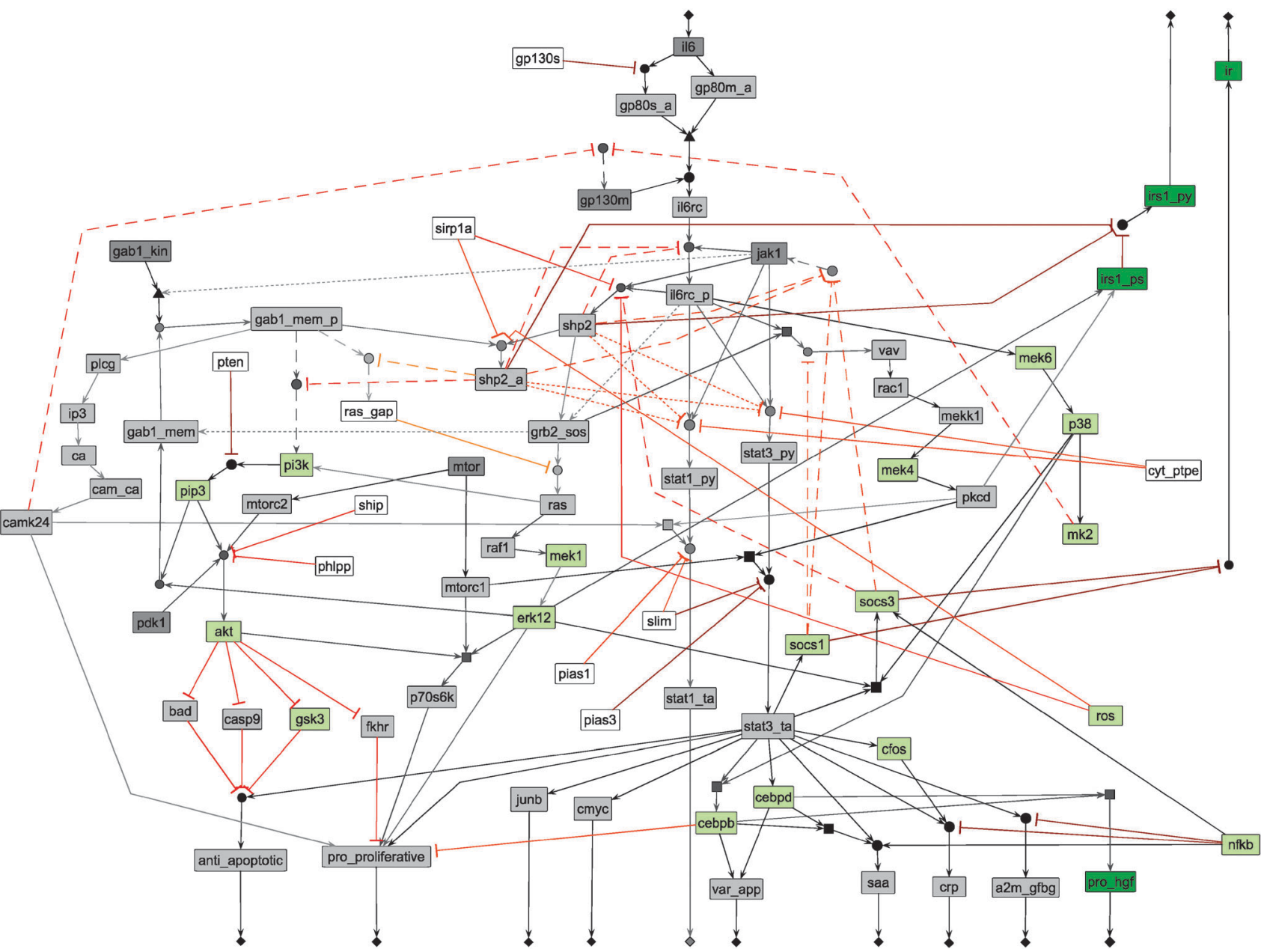

Fig. 2 Logical (master) model of IL-6 receptor signalling. See legend of Fig. 1 for further explanations.

followed by indirect up-regulation of SHP2 phosphatase activity and RasGAP release finally boosting ERK activity, uncovered an additional, classical positive FL mechanism, made up of a positive forward path and feedback (Fig. 3B, right). Therefore, the latter strategy may be regarded as "autonomous", given that no negative feedback is involved and might furthermore explain the high percentage of positive FLs (60.9\%) within the IL-6 network. Removing the IL-1-regulated species MKP1 and IL-1Ra (in IG1) or IL-6-induced SHP2 and membrane-bound phospho-Gab1 (in IG2) would break all positive FLs.

The wealth of signalling species and interactions implies complex signalling pathways where network-wide interdependencies can hardly be interpreted intuitively. By way of example, IG1 encompasses 24372 paths (half of them negative) leading from ligand IL-1 $\beta$ (input) to IL-8 expression (output). When focusing on the initial response (i.e. neglecting secondary " $\tau=2$ " events), 1386 exclusively positive paths remained. By checking out the existence of shortest positive or/and negative directed paths ${ }^{42}$ between each pair of species, we could individually specify the "triggering" effectors as activators or/and inhibitors (see also "Methods"). Revealed information on network-wide interdependencies were visualised in dependency matrices. ${ }^{31}$ The large number of ambivalent dependencies (owing to nodes that act positively and negatively on certain down-stream targets; $c f$. Fig. S3 and S5, ESI $\dagger$ ) is significantly reduced when excluding "late" edges closing feedback loops (Fig. S4 and S6 (ESI $\dagger$ ); dashed " $\tau=2$ " interactions in Fig. 1 and 2 omitted). Residual ambivalences with respect to IG1 result from: (i) involvement of normal (IL-1RI and IRAK1) as well as catalytically aberrant enzyme isoforms (IL-1RII and IRAK1c) in signal propagation, (ii) ABIN2, alternatively supporting proteasomal TRAF6 and/or NEMO degradation via A20, (iii) soluble receptor complex components (sIL-1RAcP and sIL-1RI/II) blocking activation of functional (IL-1RI) as well as non-functional (IL-1RII) receptors, (iv) IL-6 and CCL2 up-regulation by activation of transcription factors $\mathrm{AP}-1$ and $\mathrm{NF}-\kappa \mathrm{B}$ while simultaneously inhibiting required basal GSK 3 activity, and (v) IL-1 $\beta$ de novo synthesis by promoting precursor (pro-IL-1 $\beta$ ) expression, however preventing their processing due to inhibition of NALP inflammasomes. Similar effects in IG2 arise from: (i) SHP2, acting as an essential adaptor protein but also negative-regulatory phosphatase and (ii) opposing influences of transcription factors STAT3 and C/EBP $\beta$ on cell proliferation.

\section{Studying the input-output behaviour of the logical models}

Taking Boolean relationships between converging edges (or influences) explicitly into account (cf. "Methods" and supplementary model documentations, ESI $\dagger$ ) we next focused on predictions of the qualitative network response upon stimulation, 
A

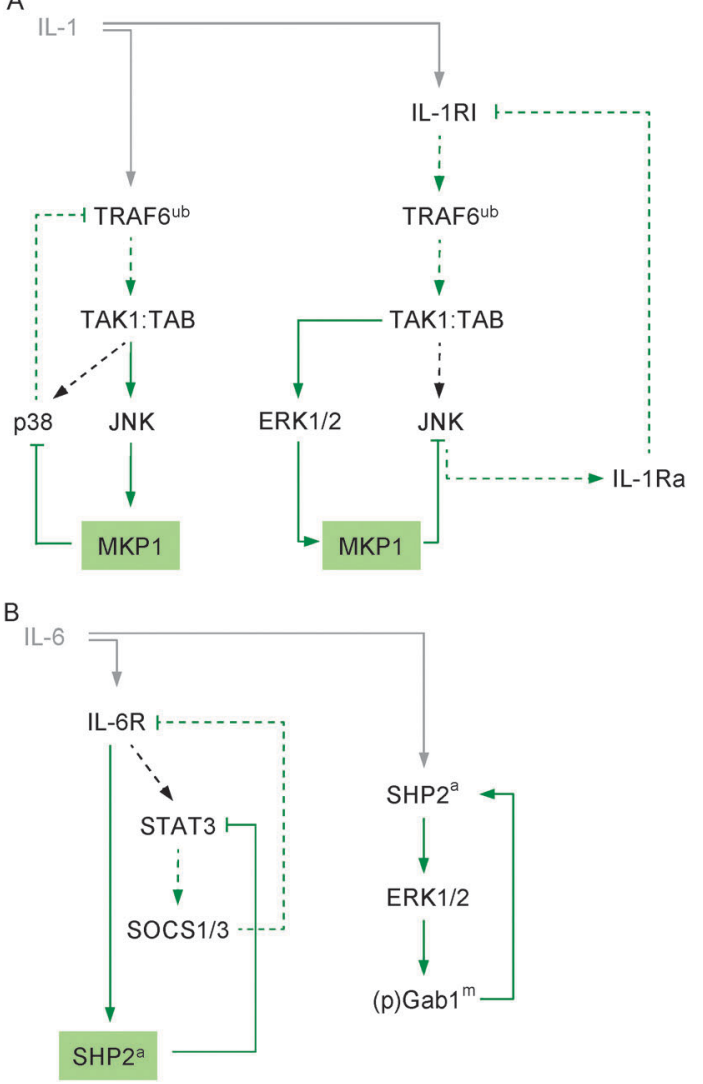

Fig. 3 Basic concepts of positive feedback loops in A: IL-1 and B: IL-6 signalling (schematic). Arrows (blunt-ended lines) indicate activations (inhibitions). Negative FLs being involved in positive feedback loops (green line colour) are indicated by dashed lines. Further explanations are given in the text. See model documentations (Tables S3.1 and S4.1, ESI $\dagger$ ) for full species names. Initial up-stream events associated to ligand binding are depicted in grey. Green highlighting stresses the analogous function of MKP1 and SHP2.

henceforth referred to as input-output $(\mathrm{I} / \mathrm{O})$ behaviour or $\mathrm{I} / \mathrm{O}$ response. Computationally, we turned our attention on the logical steady state (LSS) that follows from the logical model when prescribing external stimuli and/or systemic perturbations. The LSS and thus $\mathrm{I} / \mathrm{O}$ response of each species was calculated by propagating the preassigned binary states of input variables (ligands and "side effectors") in keeping with specified logical functions ( $c f$. "Methods" and Klamt et al. ${ }^{30,31}$ ). As already mentioned, we concentrated on the initial cellular response by excluding secondary " $\tau=2$ " events (see dashed/ dotted lines in Fig. 1 and 2). It should furthermore be underlined that a node's LSS computed for a given scenario does not depict when, but how the former responds to stimulation. The respective time range required for establishing a certain steadystate activation pattern may differ among signalling molecules attributable to intracellular localisation and kinetic parameters.

We started with " $\tau=1$ " $\mathrm{I} / \mathrm{O}$ response simulations in $\mathrm{M} 1$, thereby assuming the presence of at least one IL-1 isoform, the initial absence of SOCS proteins, and side effector states preset by default values as indicated in Fig. S7, ESI. $\uparrow$ The former provided a uniquely computable $\mathrm{I} / \mathrm{O}$ response for all species except for iNOS (Fig. S7, ESI $\dagger$ ). Due to pending scientific clarification, preventing a clear distinction between AND or OR connection of respective inputs regarding iNOS regulation, the latter was modelled using an ITT gate (see "Methods"). As both (positively contributing) inputs differ with respect to their logical states (HNF4 $\alpha$ : 1/on vs. ROS: 0/off by default) a unique $\mathrm{I} / \mathrm{O}$ response for iNOS cannot be concluded. In contrast, the other 10 ITT gates did not impede unique $\mathrm{I} / \mathrm{O}$ response determination owing to some "key" upstream effectors (e.g. MYD88, regulating IRAK1/4 and TAK 1 , contributing to $\mathrm{p} 38$, JNK, and IKK $\beta /$ ERK activation) that trigger the concurrent activation of various signalling molecules via (coherent) feed-forward loops, reflecting potential redundant pathways within the network. According to current knowledge, ${ }^{10,11,43}$ stimulation with IL-1 leads to IL-8 expression as well as SAA and LBP synthesis, whereas IL-6 and CCL2 release is down-regulated by inhibitory GSK3 serine phosphorylation. Strikingly, IL-1 $\beta$ secretion is initially blocked due to inactive caspase-activating complexes, so called NALP inflammasomes, catalysing the processing of generated pro-IL-1 $\beta$ precursors but being suppressed by ERK in response to IL-1 stimulation (Fig. S7, ESI $\dagger$ ). Closing a negative feedback loop by allowing negative-regulatory phosphatase MKP1 to inhibit causative ERK activity would, however, theoretically support post-initial IL-1 $\beta$ release, given sufficient precursor amounts. Similar initial response $(\tau=1)$ analysis in model M2 uncovered the anti-apoptotic and pro-proliferative effects of IL-6 stimulation, typically associated with proinflammatory cytokines (Fig. S8, ESI $\dagger$ ). Assuming that IL-6 does not activate NF- $\mathrm{BB}, \mathrm{M} 2$ furthermore predicts expression of acute-phase proteins (e.g. CRP, $\alpha_{2} \mathrm{M}, \gamma \mathrm{FBG}$ ) with exception of SAA which requires the co-regulation by NF- $\mathrm{KB}$ illustrating potential IL-1/IL-6 crosstalk scenarios (Fig. S8, ESI $\dagger$ ). Moreover, both models provide evidences for links between inflammation and cellular insulin resistance as well as tissue regeneration and tumourigenesis, revealing (i) inhibitory IRS1 serine phosphorylation, SHP2-mediated IRS1 inhibition or IR blocking by SOCS proteins, and (ii) hepatocyte growth factor (HGF) precursor release and its uPA-regulated processing in response to IL-1 or/and IL-6 (Fig. S7 and S8, ESI $\dagger$ ).

Systemic failure modes might disturb cellular balance and open the way for chronic inflammation and cell transformation. Using the concept of minimal intervention sets (MIS) $)^{31,37}$ we exemplarily identified support-minimal sets of species perturbations (e.g. constitutive activations or knock-outs; see also "Methods") that would provoke a specific cell response of interest. Generally, all interactions were incorporated during MIS computation, ensuring the results to be valid for primary $(\tau=1)$ as well as late or secondary events $(\tau=2)$. By calculating failure modes in M1 that would lead to permanent IL-8 expression, we could, for instance, identify 56 MISs enforcing uncontrolled, IL-1-induced or autonomous secretion of pro-inflammatory IL-8. The latter would require at least three co-existing (co-occurring) systemic faults within the network, in any case involving constitutive TAK1 (or MEKK3) and NF-KB activation. Under normal conditions, this seems very unlikely given that mentioned effector activities are strictly downregulated by internal feedback loops via MK2 and IкB $\alpha$ or, again pointing to crosstalk effects between IL-1 and IL-6, by inhibitory SOCS proteins up-regulated by IL-6. MIS studies also underlined the significance of TAK1 and/or ERK repression 
through post-initial feedback mechanisms for IL-1 $\beta$ precursor processing. Consequently, the coupling of IL-1-stimulated IL-1 de novo expression with negative feedback initiation prevents autocrine loops while still enabling intercellular signal propagation. Referring to model M2, MIS computation analogously revealed that unrestricted cell proliferation and viability in response to IL-6 is prevented with onset of negative feedback loops as SOCS expression and SHP2 phosphatase activity induce cell cycle arrest. Accordingly, inactivation of SOCS or of SHP2 represent failure modes with respect to permanent pro-proliferative effects in response to IL-6 stimulation (i.e. pro-proliferative $=1$ for $i l 6 \equiv 1$ ).

In the light of MIS analysis results, both modelled signalling networks comprise intrinsic safety mechanisms in the form of negative feedback loops counteracting potential, e.g. mutational, alterations of effector activities and thereby supporting systemic robustness.

\section{Hepatocellular validation of the models by integrating experimental data}

Structural network analysis of the models provided insights into general network properties of IL-1 and IL-6 signalling and may, e.g. by MIS analysis, offer a platform for studying pathophysiological behaviours and for identifying potential drug targets. However, as these "master" models were constructed from literature data covering several cell types, they are not implicitly applicable to hepatic conditions. In order to uncover hepatocellular characteristics, we compared model predictions to high-throughput experimental data assayed in primary human hepatocytes for $t=0$ and $30 \mathrm{~min}$ by Alexopoulos et $\mathrm{al}^{32}$ As introduced previously by Samaga et al..$^{30}$ we (i) verified the interaction graphs by contrasting raw data trends with corresponding dependency matrix predictions and (ii) looked for logical misinterpretations uncovered by inconsistencies between discretised data and network $\mathrm{I} / \mathrm{O}$ responses simulated according to experimental conditions using the logical models. Detailed descriptions on experimental data and analysis procedures are given in "Methods" and Tables S1 and S2 (ESI + ); raw data sets and transformed data applied for IG1/M1 and IG2/M2 validation are visualised in Fig. S9 and S10, ESI. $\dagger$

\section{Data analysis by means of interaction graphs}

According to procedures explained in "Methods" and using the readouts shown in Fig. S9.A and S10.A (ESI $\dagger$ ) we first verified the models for IL-1 and IL-6 signalling by focusing on the underlying interaction graphs IG1 and IG2, respectively. Given the measurement time range $t=0-30$ min primarily encompassing initial response events, we again excluded " $\tau=2$ " interactions when calculating corresponding dependency matrices.

As generally observed, measured trends in species phosphorylation in response to certain stimulus scenarios could largely be mapped by suggested network topologies. Regarding IG1 (IG2), 78\% (80\%) of calculated species dependencies agreed with data trends: phosphorylation levels increased in $31 \%(30 \%)$ and decreased in $7 \%(9 \%)$ of the analysed cases according to predictions; in $40 \%(41 \%)$ of all regarded cases an effect was neither detected in the measurements nor expected with respect to pathway topologies. In $10 \%(9 \%)$ of all cases a significantly increasing or decreasing phosphorylation level was found experimentally though no effects had been expected from topology. The remaining $12 \%(11 \%)$ of the cases ( $c f$. grey entries in Fig. 4 and 5) indicate model-predicted positive or negative effects not significantly evident in experimental readouts. Those "grey" cases may not represent a model falsification as perturbation of the start node of a path does not necessarily induce a significant effect on the activity of the target (end) species, e.g. owing to saturation effects or experimental assay detection limits. Fig. 4 and 5 depict consistencies and discrepancies in detail. Conclusions with respect to model modifications are discussed below.

\section{Data analysis using the logical models}

For verifying predictions of the Boolean models regarding the initial IL-1- or IL-6-induced response behaviour, we converted continuous raw data, individually assayed for a predefined treatment scenario, into binary signal curves (mapping species activation levels to $0 /$ off or $1 /$ on $)$ and compared them to simulated initial $\mathrm{I} / \mathrm{O}$ responses in $\mathrm{M} 1$ and $\mathrm{M} 2$ with strict respect to experimental settings (see "Methods" for further descriptions). We again concentrated on initial post-receptor events and further assumed "general case" cellular signal propagations by omitting all " $\tau=2$ " interactions.

$87 \%$ of all tested species activation levels regarding IL-1-associated treatment scenarios could be successfully reproduced by M1. The additional omission of inhibitordissociation processes initiated through MEKK3 activation, alternatively contributing to I $\mathrm{K} \mathrm{\beta} \alpha$ release from transcription factor NF- $\mathrm{KB}$ (as proposed by Yao et al. ${ }^{44}$ ), increased model consistency to $88 \%$ (Fig. 6). One might therefore conclude that $\mathrm{I} \kappa \mathrm{B} \alpha$ inhibition is, at least in initially responding primary human hepatocytes, predominantly mediated through degradation, whereas dissociation seems negligible.

Simulation of IL-6-induced signalling events in M2 revealed $82 \%$ conformance of model predictions and discretised experimental data, leaving inconsistencies primarily attributable to p70S6K activation $(9 \%)$ integrated via ITT gate expression and thus provoking indeterminable states $(c f$. yellow entries in Fig. 7). Again, conclusions with respect to model modifications are discussed below.

\section{Interpretation of discrepancies between model predictions and measured data and hepatocellular model optimisation}

The detected inconsistencies between experimental data and model predictions regarding the initial cellular response upon interleukin and/or inhibitor treatment of hepatocytes revealed suitable starting points for optimisation and specification of the introduced "master models" with respect to hepatocytic characteristics. Referring to the delineated discrepancies concerning predicted and measured responses (Fig. 4-7), the following model modifications, starting with model M1, were introduced to improve consistency.

- Positive-regulatory influence of IKK $\beta$ on Akt activity: the yet missing but experimentally evident positive-regulatory influence of IKK $\beta$ on Akt activity ( $c f$. Fig. 4 column 1; line 5 


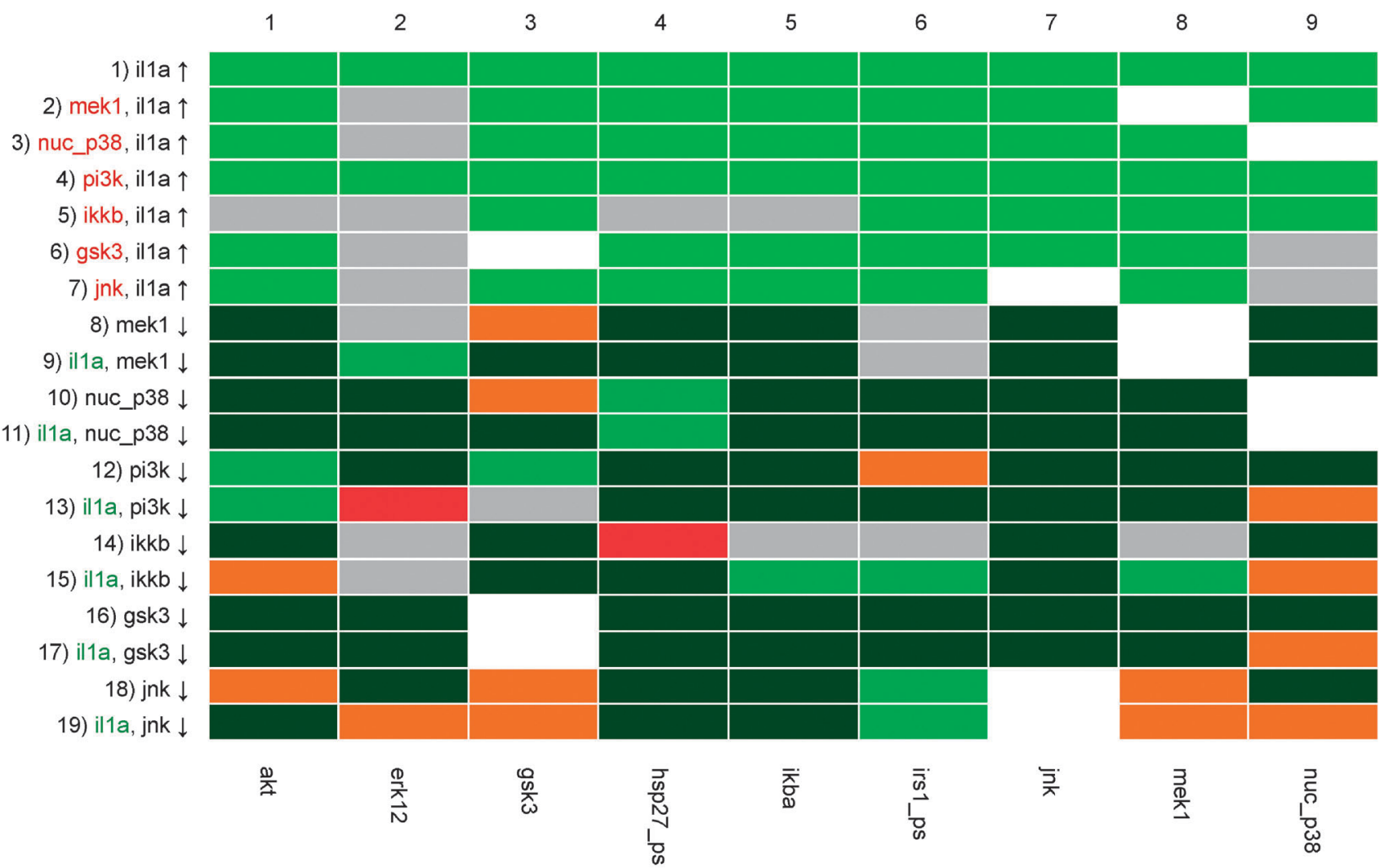

Treatment effects on respective species phosphorylation in view of model predictions:

$\square$ increase, as expected
decrease, as expected
no effect, as expected

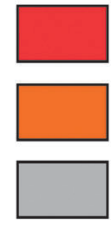

increase, though no effect expected

decrease, though no effect expected

no significant effect, though positive or negative one expected

effect on a measured species not considered if the latter is a direct target of the applied inhibitor

Fig. 4 Comparison of interaction graph-based predictions and measured responses for various stimulus-response experiments with IL-1 $\alpha$ in primary hepatocytes. Measured effects on species activation levels for $t=30 \mathrm{~min}$ caused by different treatment scenarios (ligand and/or inhibitor) in primary human hepatocytes (data published by Alexopoulos et al. ${ }^{32}$ ) were compared to predicted species dependencies within IG1 subject to primary events. Each row represents the comparison of two experimental treatment scenarios $\left(S_{1}\right.$ and $\left.S_{2}\right)$ to qualify corresponding effects on network species (readouts) listed in the columns. Row labels are as follows: green (red) font colour indicates cytokine (inhibitor) application in both scenarios $S_{1}$ and $S_{2}$. Up (down) arrows attached to species with black font colour mark cytokine (inhibitor) application in scenario $S_{1}$, implicating no cytokine or inhibitor addition in $\mathrm{S}_{2}$. For example, row 9 depicts the results when comparing $\mathrm{S}_{1}$ (IL-1 $\alpha$ ligand + MEK1 inhibitor) with $\mathrm{S}_{2}$ (IL-1 $\alpha$ ligand), therefore showing effects of an increasing MEK1 inhibitor level in the presence of IL-1 $\alpha$ on the readouts in the columns. Matrix elements indicate structural (in-)consistencies according to the given colour legend. See "Methods" and Fig. S9.A (ESI $\dagger$ ) for further information and raw data. Negative states: gsk3, ikba (see "Methods").

and $15(1 ; 5 / 15)$ and column 1; line 5 (1;5) in Fig. 5) was integrated indirectly by assuming ERK1/2-mediated p90RSK activation, subsequent p90RSK- or directly ERK-regulated Raptor ( $\rightarrow$ mTORC1) activation, ${ }^{45,46}$ mTORC1-mediated p70S6K activation, ${ }^{23,47}$ and up-regulation of catalytic Rictor ( $\rightarrow$ mTORC2) activity by p70S6K, ${ }^{48,49}$ the former being directly involved in Akt activation. ${ }^{50}$ We therefore expanded the IL-1-associated signalling network by three additional species - mTORC1, p70S6K, and p90RSK - the latter two seeming verifiably active upon IL-1 stimulation of primary human hepatocytes. ${ }^{32}$

- Positive influence of JNK on MEK1 and ERK activity: to reproduce the positive influence of JNK on MEK1 and ERK activity (see $(8 ; 18 / 19)$ and $(2 ; 19)$ in Fig. 4 and $(2 ; 14)$ in Fig. 6), we contemplate an alternative, redundant and IKK $\beta$-independent (cf. $(8 ; 12)$ in Fig. 6) MEK1 activation mechanism via JNK more significantly contributing to (apparently weak) ERK than to MEK1 activation (see $(8 ; 14)$ in Fig. 6), though yet 


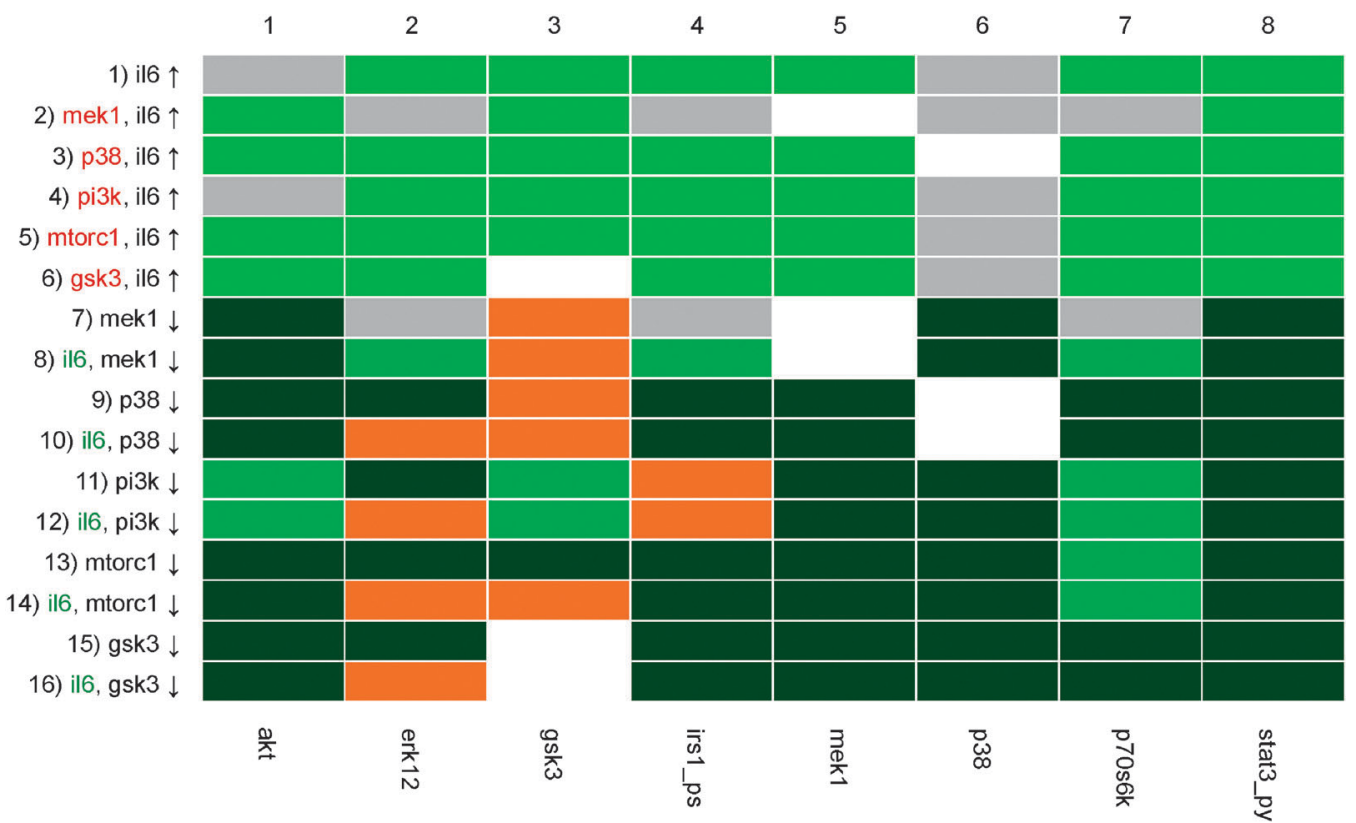

Fig. 5 Comparison of interaction graph-based predictions and measured responses for various stimulus-response experiments with IL-6 in primary hepatocytes. See Fig. 4 for further explanations and colour legend. Predictions were made using IG2 subject to primary events. Respective raw data are given in Fig. S10.A (ESI †). Negative state: gsk3 ( $c f$. "Methods").

lacking any biochemical confirmation. Generally, modifications concerning IL-1-induced ERK activation are stated under reserve, given measured ERK phosphorylation levels close to or even beneath technical detection limits (see Fig. S9.A, ESI $\dagger$ ), partially impeding the falsification of already integrated interactions ( $c f$. (2; grey elements) in Fig. 4). A positive link between JNK and MEK1/ERK activity would furthermore explain the at least basally significant up-regulation of Akt by JNK (see $(1 ; 18)$ in Fig. 4). Nevertheless and with respect to inconsistencies apparent without additional IL-1 application ( $c f$. row 18 in Fig. 4), unspecificity of the applied JNK inhibitor (Table S2, ESI $\dagger$ ) might qualify all given statements concerning additional JNK involvement.

- Positive-regulatory influence of Akt on p38 MAPK activity: given the above mentioned suggestions for integration of an 'IKK $\beta \rightarrow$ Akt' and 'JNK $\rightarrow$ ERK1/2' (thus indirect 'JNK $\rightarrow$ $\mathrm{Akt}$ ') interdependence, the additional introduction of an alternative positive-regulatory influence of Akt on p38 MAPK activity seems to be a convenient, though not yet documented way to simultaneously cover the respective JNK, IKK $\beta$, and PI3K involvement (see $(9 ; 13 / 15 / 19)$ in Fig. 4 and $(9 ; 14)$ in Fig. 6). However, we suggest this with reservations, given the abundance of discrepancies related to IL-1-induced p38 MAPK regulation ( $c f$. column 9 in Fig. 4), which might also point to systematic uncertainties owing to marginal signal strengths (Fig. S9.A, ESI $\dagger$ ). Apart from that, cellular stress effects mediated by 38 MAPK cannot be ruled out.

- Positive influence of p70S6K and basal effects of IKK $\beta$ on phospho-Hsp27-S78: IKK $\beta$ appears to basally repress Hsp27-S78 phosphorylation (see $(4 ; 14)$ in Fig. 4) - an effect that might get superimposed upon IL-1 stimulation $(c f .(4 ; 15)$ in Fig. 4) due to positive-regulatory p38/MK2 onset ${ }^{51}$ (correlation already integrated) or alternative, direct p70S6K contribution. The latter, being activated in primary human hepatocytes in response to IL- $1,{ }^{32}$ has been shown to act as a potential Hsp27-S78 kinase in vivo. ${ }^{52}$ Introducing an additional 'p70S6K $\rightarrow$ Hsp27-pS' link would further facilitate the reproduction of p38-independent phospho-Hsp27-S78, apparent despite p38 knock-down (see $(4 ; 10)$ in Fig. 6). Referring to the logical inconsistency highlighted by $(4 ; 12)$ in Fig. 6, one should note that IL-1 induces similar signal strengths for $t=30$ min concerning Hsp27-S78 phosphorylation regardless of IKK $\beta$-inhibitor application ( $c f$. Fig. S9.A (ESI $\dagger)$, right). However, inhibitor-associated loss of suggested basal repression dramatically increases the phosphorylation level for $t=0$ min, hence lowering the activating "net-effect" caused by ligand add-on (see Fig. S9.A (ESI $\dagger$ ), right) eventually used for verification of logical implementations but not detectable by the predefined parameter set in this special case $($ see $(4 ; 12)$ in Fig. S12, ESI $\dagger$ ). Due to ambivalences occurring when additionally integrating inhibitory basal IKK $\beta$ effects on Hsp27-pS (Fig. 8), the latter were excluded during IG1 optimisation, though maintaining the topological discrepancy $(c f .(4 ; 14)$ in Fig. S11, ESI $\dagger$ ).

- GSK3 inhibition: as the observed IL-1-induced effects on GSK3-S9 phosphorylation (irrespective of inhibitor application) range close to threshold parameters (see "Methods") and are therefore not detected in the course of discretisation (see $(3 ; 8)$ in Fig. 6), we do not speculate on alternative or cooperative inhibitory GSK3 regulation mechanisms. Nonetheless, studies by Ding et al. ${ }^{53}$ focusing on IGF-1- and HBX-stimulated HepG2 or HepB3 cells, unveiled a positiveregulatory influence of ERK-dependent p90RSK activity on phospho-S GSK3, generally seeming more relevant in vivo than a respective p70S6K contribution. ${ }^{54}$ Given that both kinases, p90RSK and p70S6K, seem to be up-regulated in response to IL-1 (see above), mentioned effects should be kept in mind. 


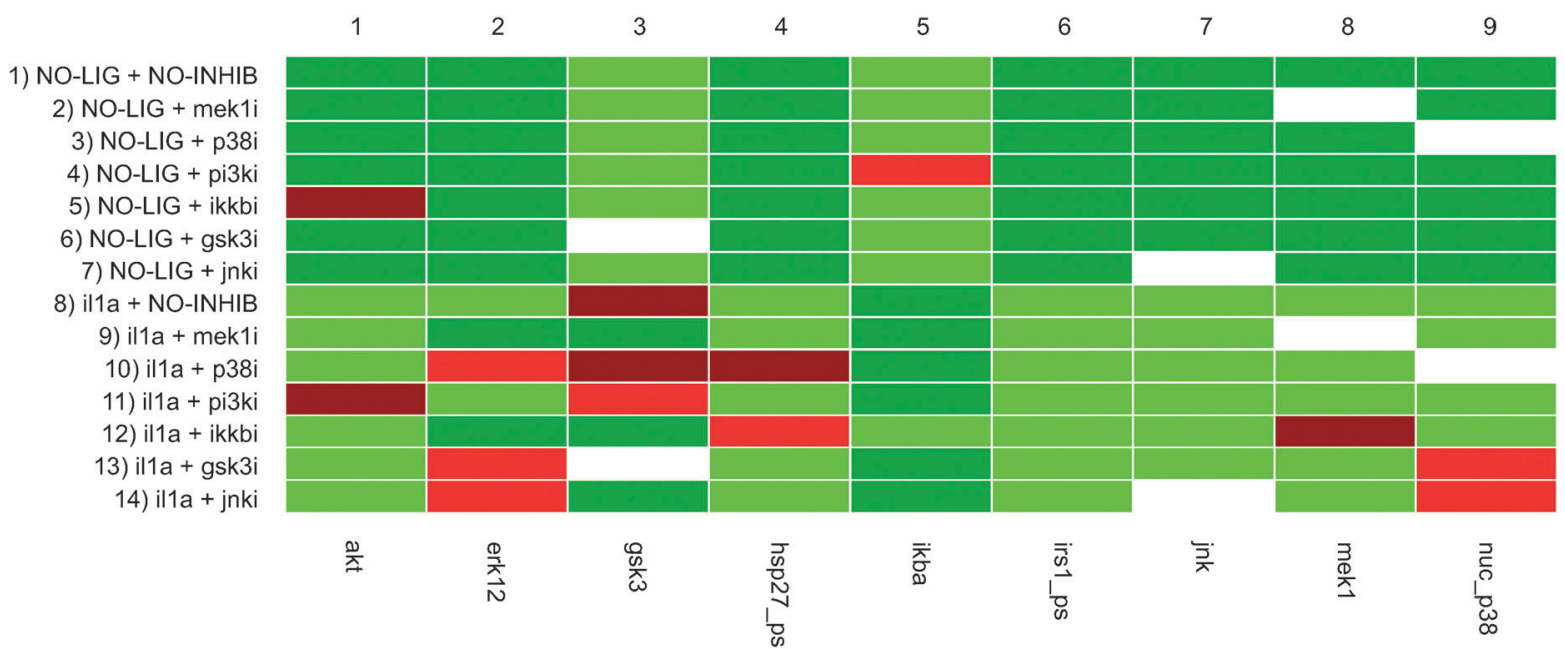

Species activation ...

predicted correctly, "on"

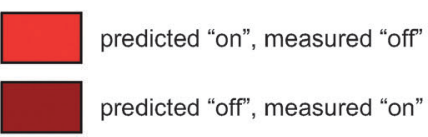

predicted correctly, "off"

predicted "off", measured "on"

effect on a measured species not considered if the latter is a direct target of the applied inhibitor

Fig. 6 Comparison of measured responses and predictions from the logical IL-1 model for various stimulus-response experiments with IL-1 $\alpha$ in primary hepatocytes. Discretised activation levels (raw data assayed in primary human hepatocytes; $c f$. Alexopoulos $e t$ al. ${ }^{32}$ Fig. S9.B (ESI $\dagger$ ), and "Methods") for $t=30$ min were checked against respective logical I/O responses computed using model M1 subject to primary events. Each row refers to a particular treatment scenario $(\mathrm{A}+\mathrm{B})$ indicated on the left hand side. Respective readouts are given in the columns. Matrix elements outline accordances or discrepancies between measured and predicted responses in keeping with colour legend. See text and "Methods" for further information. Negative states: gsk3, ikba. Abbreviations: NO-LIG: no ligand/negative control; NO-INHIB: no inhibitor. Respective raw data are given in Fig. S9.A (ESI $\dagger$ ).

Structural modifications covering the described interdependencies and summarised in Fig. 8 increased logical consistency in $\mathrm{M} 1$ to $89 \%$ (Fig. S12, ESI $\dagger$ ). Topological contradictions with respect to IG1 underlying IL-1 signalling were reduced to $3 \%$ (from $10 \%$ ), though raising the percentage of experimentally insignificant effects (grey entries) to $25 \%$ (from $12 \%$ ) and thereby lessening exact agreements to $72 \%$ (from $78 \%$; Fig. S11, ESI $\dagger$ ). However, one should contemplate that, owing to parallel pathways and saturation effects, signal transduction along a positive path may not obligatorily lead to significant up-regulation of its target species and is therefore not explicitly quantifiable during measurements. This aspect might explain the higher number of grey entries regarding the "optimised" IL-1 signalling network. Nonetheless, handling such marginal cases a priori seems to be of secondary importance compared to reducing the number of definitely falsified predictions

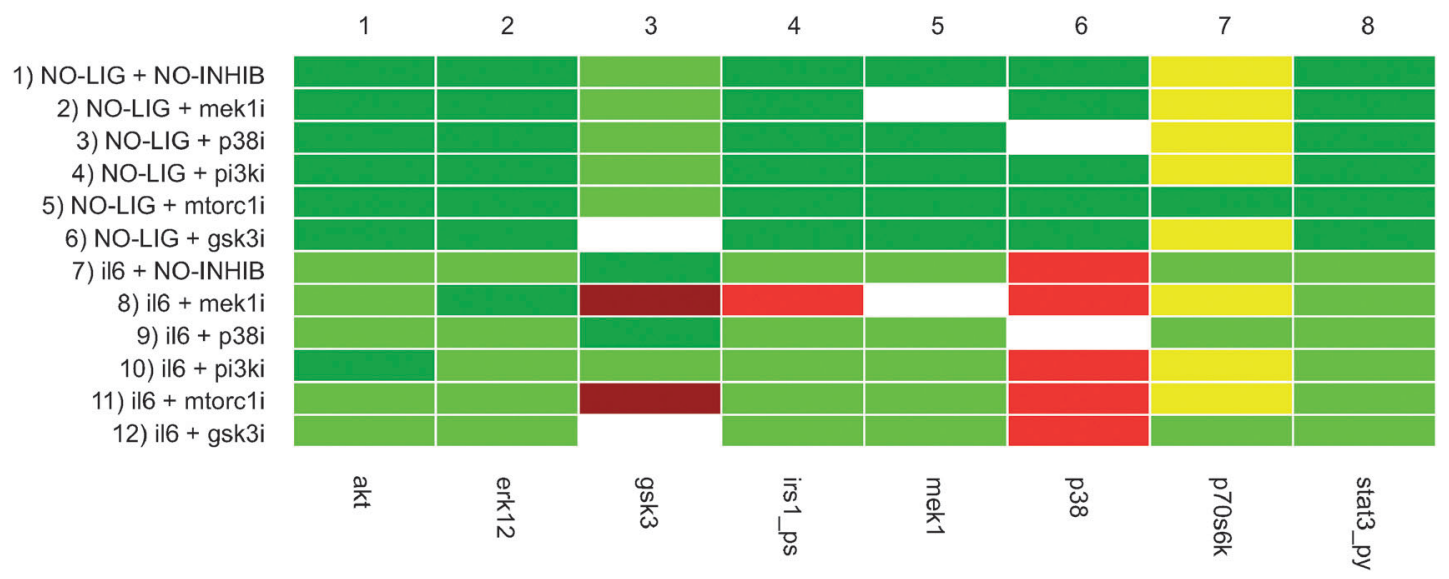

Fig. 7 Comparison of measured responses and predictions from the logical IL-6 model for various stimulus-response experiments with IL-6 in primary hepatocytes. See Fig. 6 for further explanations and colour legend. Analysis was performed using the discretised activation levels depicted in Fig. S10.B (ESI $\dagger$ ) and model M2 subject to primary events. Yellow matrix elements outline model inconsistencies related to used ITT gate expressions, the latter causing indeterminable states during simulation. Negative state: gsk3. Respective raw data are given in Fig. S10.A (ESI $\dagger$ ). 


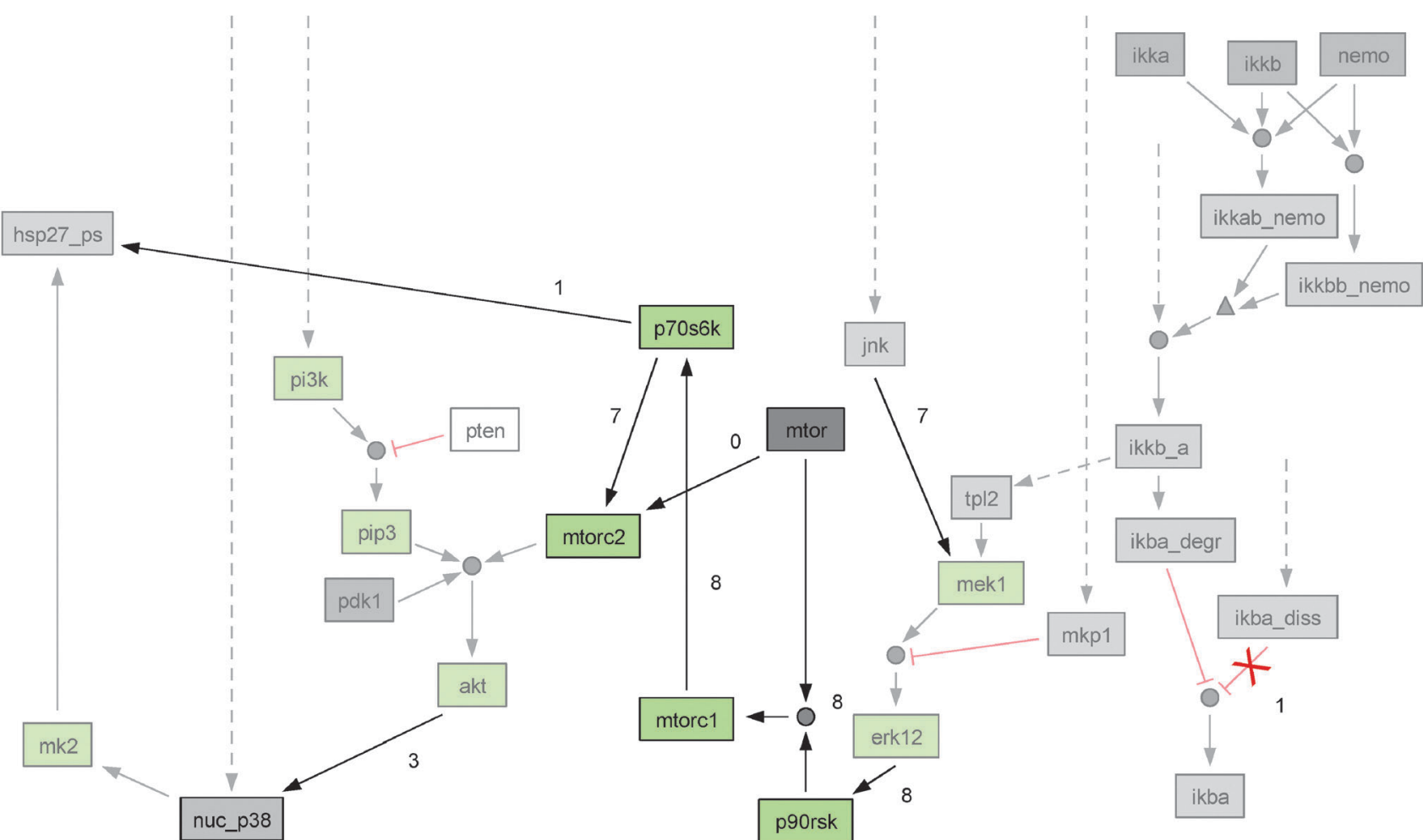

Fig. 8 Network modifications referring to initial IL-1 receptor signalling in primary human hepatocytes. See Fig. 1 and "Methods" for further information on symbols etc. Species and interactions not directly affected as well as confidence levels are not depicted for reasons of clarity. Faint components are adopted from master model representation with dashed arrows outlining up-stream activation events (see Fig. 1). Newly introduced effectors and reactions are coloured intensely. Interactions seeming negligible with respect to initial cellular response according to our data analysis results are crossed out. Numbers at new/to be removed edges show the respective interaction's level of evidence, i.e. the numerical increase of wrong predictions (red/orange entries) in Fig. S11 and S12 (ESI $\dagger$ ) when removing the modification from the optimised network (see text for further explanations).

(red/orange entries in Fig. 4-7). Remaining discrepancies (Fig. S12, ESI $\dagger$ ) may give rise to additional clarifications concerning the roles of PI3K or GSK3 in IL-1-induced ERK or p38 MAPK activation, respectively $(c f .(2 ; 13)$ and $(9 ; 17)$ in Fig. S11, ESI $\dagger$ ), and considerations on a possible, basally relevant positive effect of endogenous PI3K on IRS1-S phosphorylation (see $(6 ; 12)$ in Fig. S11, ESI $\dagger$ ) in hepatocytes.

To quantify the overall improvement of the model we introduce two simple error scores. The absolute error score $\left(E_{\text {abs }}\right)$ represents the total number of wrong predictions, i.e. the number of red/orange entries in Fig. 4 (wrong interactiongraph based predictions) plus those in Fig. 6 (wrong predictions from the logical model). The relative error score $\left(E_{\text {rel }}\right)$ results from the absolute error score divided by the total number of predictions made. Regarding the original model M1, we get $E_{\mathrm{abs}}^{\mathrm{M} 1}=30$ and $E_{\mathrm{rel}}^{\mathrm{M} 1}=10.8 \%$ (with 277 predictions from Fig. 4 and 6). With respect to network modifications shown in Fig. 8, the optimised model M1 yields error scores of $E_{\mathrm{abs}}^{\mathrm{M} 1 \mathrm{opt}}=$ 18 and $E_{\mathrm{rel}}^{\mathrm{Mlopt}}=6.5 \%$ (Fig. S11 and S12, ESI $\dagger$ ) reflecting a $40 \%$ error reduction. These scores also enabled us to quantify the relative contribution (or evidence) of the introduced network changes. Taking the optimised model as a basis, we individually removed each modification $m$, recomputed $E_{\mathrm{abs}}^{\mathrm{Mlopt}-m}$ and defined the increases in the number of wrong predictions, i.e. the difference $d_{\mathrm{m}}=E_{\mathrm{abs}}^{\mathrm{Mlopt}-m}-E_{\mathrm{abs}}^{\mathrm{Mlopt}}$, as the evidence level of modification $m$. The evidence levels of all modifications in M1 are shown as (edge) weights in Fig. 8. For example, one can see that modifications related to p90RSK, mTORC1, and p70S6K had the strongest impact on model improvement. The introduced edge from the reservoir variable 'mtor' to 'mtorc2' did not affect the error score. However, 'mtor' was nonetheless included to have a biologically reasonable description consistent to that used within the IL-6 network.

Corrective modifications referring to IL-6 signalling (represented by model M2) were realised as follows.

- IL-6-induced p38 MAPK activation seems negligible in hepatocytes, given indistinguishable effects of ligand application on phosphoproteomic signal strength (see $(6 ; 1-6)$ in Fig. 5 and $(6 ; 7-12)$ in Fig. 7; and Fig. S10.A (ESI $\dagger$ ) depicting p38 MAPK activation below detection limit (grey background)). Nevertheless, it has to be clarified how insignificantly up-regulated p38 MAPK positively (though likely redundantly; $c f$. respective entries in Fig. 7) contributes to ERK activation and inhibitory GSK3-S phosphorylation (see $(2 ; 10)$ and $(3 ; 9 / 10)$ in Fig. 5) upon IL-6 stimulation.

- ERK up-regulates p70S6K via mTORC1 activation/no Akt involved: as already used to optimise the IL-1 signalling network (see above), we imported the scenario (hitherto undocumented for hepatocytes) delineated by Carriere $e t$ al. whereupon ERK targets and phosphorylates the mTOR scaffolding protein Raptor $(\rightarrow$ mTORC1) on several serine residues either directly ${ }^{46}$ or via p90RSK activation,${ }^{45}$ boosting its activity. 
Documented p90RSK activation by primary human hepatocytes in response to IL- ${ }^{32}$ may support this hypothesis. According to statements by $\mathrm{Kim}^{23}$ and Shi et al., ${ }^{47} \mathrm{mTORC1}$ might subsequently mediate p70S6K-S phosphorylation, highlighting an Akt-independent activation mechanism. Re-emerging discrepancies regarding p70S6K regulation (see $(7 ; 8 / 11)$ in Fig. S14, ESI $\dagger$ ) seem rather attributable to yet missing, alternative MEK1/mTORC1-independent pathways than to Akt exclusion, given a possible Akt involvement by mTOR de-repression as reviewed by Hay and Sonenberg ${ }^{55}$ - an effect that would again support mTORC1 activation and therefore MEK1/mTORC1mediated p70S6K up-regulation. A positive-regulatory link between p70S6K and mTORC2 (as adopted for hepatocellular IL-1 signalling; see above and Fig. 8) in response to IL-6 has not yet been taken into account, owing to non-existent hints from data analysis concerning the associated positive influence of mTORC1 on Akt activity ( $c f .(1 ; 14)$ in Fig. S13, ESI $\dagger)$.

- IRS1-S phosphorylation by ERK vs. PKC $\delta$ : missing positive-regulatory influences of PI3K on ERK activity and subsequent ERK-catalysed IRS1-S636/639 phosphorylation (see $(2 ; 12)$ and $(4 ; 11 / 12)$ in Fig. 5) could be ascribed to the signal amplifying and Gabl-associated positive feedback loop (see above) described by Eulenfeld and Schaper. ${ }^{56}$ Referring to studies in IL-6-treated HepG2 cells by Kim et al., ${ }^{23}$ another ERK-mediated IRS1-S phosphorylation scenario, supposing p70S6K as the predominant executing kinase could be initially ruled out, given no significant impact of mTORC1 inhibition on phospho-IRS1-S636/639, though on p70S6K activity (cf. (4;13/14) and (7;13/14) in Fig. 5). Furthermore, MEK1 knock-down leading to reduced ERK activity seems sufficient to block inhibitory IRS1-S phosphorylation upon IL-6 treatment (see $(4 ; 8)$ in Fig. 7), hence revealing respective $\mathrm{PKC} \delta$ influences to be secondary.

- p70S6K contributes to inhibitory GSK3-S phosphorylation: though challenged in vivo by Stambolic and Woodgett ${ }^{54}$ and ruled out regarding initial IL-1 signalling (see above), the integration of additional p70S6K-catalysed (apart from Akt-mediated) GSK3-S phosphorylation upon IL-6 treatment removed MEK1- and mTORC1-associated inconsistencies concerning GSK3 inhibition (see $(3 ; 7 / 8 / 14)$ in Fig. 5 and $(3 ; 8 / 11)$ in Fig. 7) and might thus, also with respect to stronger ERK activation in response to IL-6 than to IL-1 ( $c f$. Fig. S9.A and S10.A (ESI $\dagger$ ), left), be relevant for hepatocytic IL-6 signalling.

Taking these changes (visualised in Fig. 9) into account, we could increase the accuracy of the logical model M2 from $82 \%$ to $98 \%$, getting residual discrepancies in just $2 \%$ of simulated scenarios attributable to pending add-ons concerning p70S6K regulation (Fig. S14, ESI $\dagger$ ). Topological accordance with respect to IG2 increased to $84 \%$ (originally $80 \%$ ), leaving $8 \%(11 \%)$ experimentally indiscernible effects (grey matrix elements) and $8 \%(9 \%)$ mismatches (Fig. S13, ESI $\dagger$ ), the latter (i) stressing the relevance for initial integration of Gab1-mediated positive-regulatory feedback events demonstrated by Eulenfeld and Schaper $^{56}$ (see respective entries of row 11 and 12 in Fig. S13, ESI $\dagger$ ), (ii) pointing to need for clarification regarding the role of p38 MAPK in IL-6 signalling ( $c f$. respective entries of row 9 and 10 in Fig. S13, ESI $\dagger$ ), and (iii) highlighting alternative, IL-6-induced ERK activation strategies somehow comprising mTORC1 and GSK3 (see $(2 ; 14 / 16)$ in Fig. S13, ESI $\dagger$ ).

Similarly as for M1, we quantified the overall improvements of model M2 by the error scores introduced above. The absolute number of errors decreased from $E_{\mathrm{abs}}^{\mathrm{M} 2}=27$ to $E_{\mathrm{abs}}^{\mathrm{M} 2 \mathrm{opt}}=12$ and relatively from $E_{\mathrm{rel}}^{\mathrm{M} 2}=12,9 \%$ to $E_{\mathrm{rel}}^{\mathrm{M} 2 \mathrm{opt}}=$ $5.7 \%$ ( $c f$. Fig. 5 and 7 vs. Fig. S13 and S14, ESI $\dagger$ ). Thus, the inaccuracy of the model was reduced by more than $50 \%$. The individual evidence levels of the modifications (Fig. 9; for definition see above) indicate that, analogously to the IL-1 network, the introduced pathway from p90RSK via mTORC1 to p70S6K had the strongest effect on model improvement.

Regarding stated model modifications, it should be pointed out that many regulatory events, though a priori regarded directly, may also reflect indirect effects transduced via several

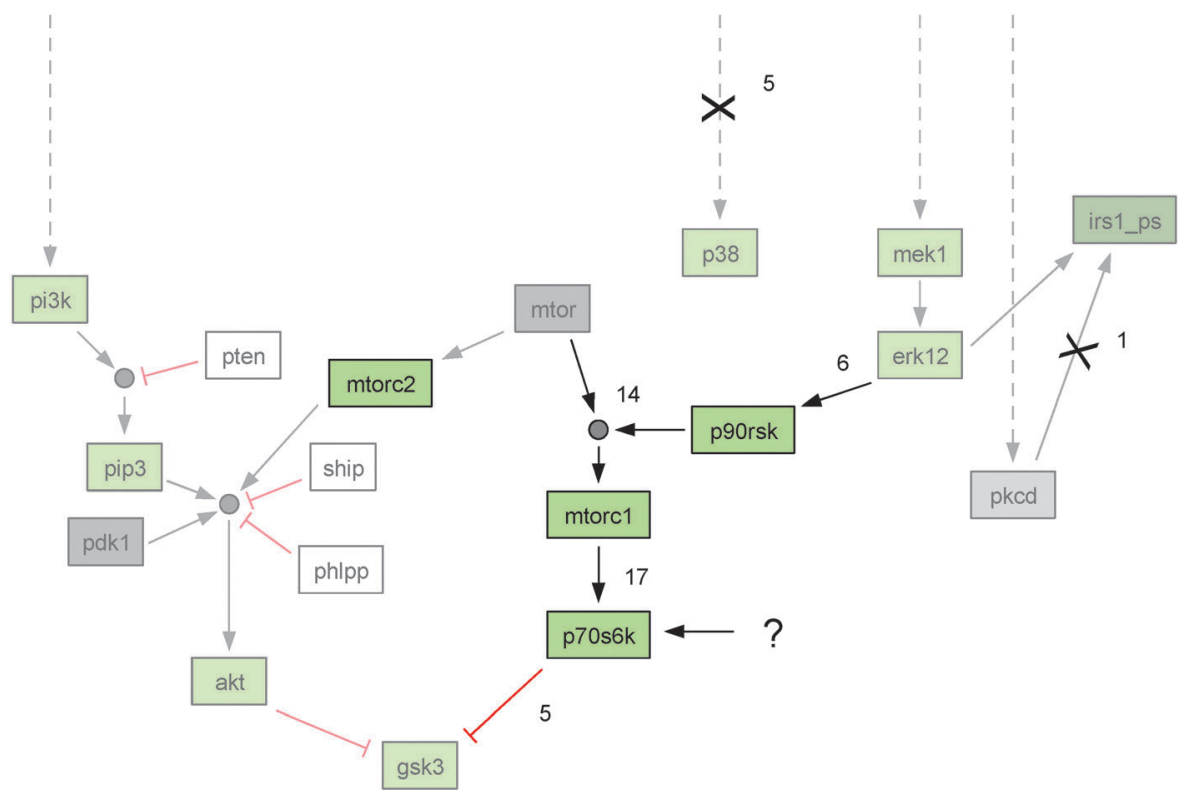

Fig. 9 Network modifications referring to initial IL-6 receptor signalling in primary human hepatocytes. See Fig. 8 and 2 for further explanations. So far unresolved mechanisms concerning IL-6-induced p70S6K activation are indicated by a question mark. 
effectors and frequently additive mechanisms (e.g. de-repression $v s$. catalytic activation) finally causing the observed "overall effects".

\section{Sensitivity of model predictions with respect to discretisation parameters}

To compare quantitative measurements with discrete predictions from our models we had to discretise the experimental data via four thresholds (one for detecting significant signal changes; three for distinguishing active from inactive states; see "Methods"). To estimate the sensitivity of the obtained results (i.e. the agreements and discrepancies in Fig. 4-7) with respect to the relevant discretisation parameters, we computed the relative number of (colour) changes in Fig. 4-7 resulting when (a) decreasing and (b) increasing all thresholds by $10 \%$ (note that the detection limit $p_{3}=500$, representing a devicedependent parameter, was not changed). For IL-1, $6.5 \%$ $(5.8 \%)$ of the entries in Fig. 4 and 6 changed colour with threshold decrease (increase); concerning IL-6 we got $8.1 \%$ $(6.1 \%)$ colour changes in Fig. 5 and 7 . This reflects a reasonable robustness of the results which is further supported by the fact that most changes concern green-to-grey or grey-togreen transitions in Fig. 4 and 5 (only few of the critical red/orange entries would disappear or newly appear). However, some discretised data points might range close to the edge between significant/non-significant effects or active/inactive states, respectively.
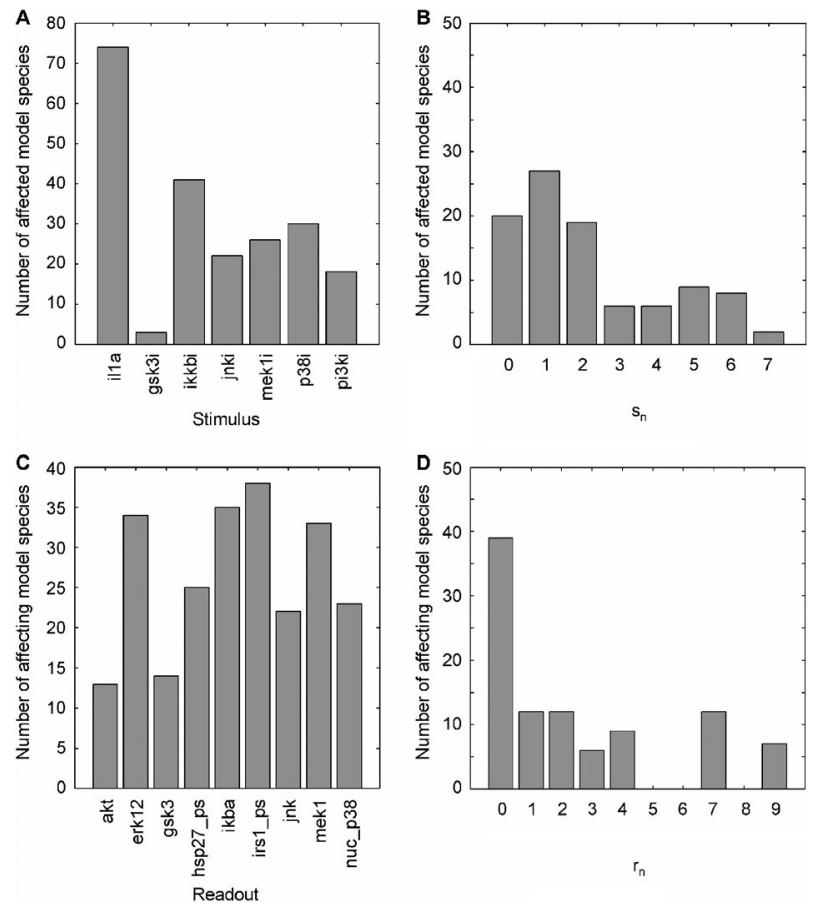

Fig. 10 Experimental coverage of modelled IL-1 receptor signalling. A: Number of network species affected by each of the applied cytokine/inhibitor treatments (stimulus). B: Distribution of numbers of perturbations affecting IL-1 signalling species. C: Number of network species affecting phosphoproteomic readouts. D: Distribution of numbers of readouts affected by IL-1 signalling species. Corresponding dependency matrices are shown in Fig. S15.A and S16.A (ESI $\dagger$ ). (Total number of IL-1-associated species: 97.)

\section{Relative network coverage with respect to perturbations and readouts}

A final issue to be discussed in conjunction with the networkbased data analysis is the coverage of the two networks with respect to (i) perturbations (i.e. how many nodes are affected by the inhibitors/cytokines) and (ii) readouts (i.e. how many nodes are directly or indirectly measured by the readouts). In order to quantify these two types of coverage, we computed for each node $n$ (separately for each model) how many of the used stimuli (IL-1/IL-6) and inhibitors (mek1i, p38i, pi3ki, mtorci, gsk3i, ikkbi, jnki; $c f$. Tables $\mathrm{S} 1$ and $\mathrm{S} 2$, ESI $\dagger$ ) had an influence on it (this number is denoted by $s_{n}$ ) and how many of the readouts are affected by it (and do thus at least partially reflect the state of $n$; this number is denoted by $r_{n}$ ). Hence, $s_{n}$ quantifies controllability and $r_{n}$ observability of node $n$. One cytokine and 6 inhibitors were used for perturbing the IL-1 network with its 97 nodes giving a maximal value of 7 for $s_{n}$ whereas the maximal number for $r_{n}$ is 9 (total number of readouts). Fig. S15.A and S16.A (ESI $\dagger$ ) depict $s_{n}$ and $r_{n}$ for each node in the IL-1 network for the primary (" $\tau=1$ ") events and Fig. 10 shows the resulting distributions of $s_{n}$ and $r_{n}$. For 16 of the network's inputs we obviously got $s_{n}=0$ except for the stimulus IL- $1 \alpha$ itself. For the other species, at least one stimulus reaches each node and some nodes are affected by five, six or
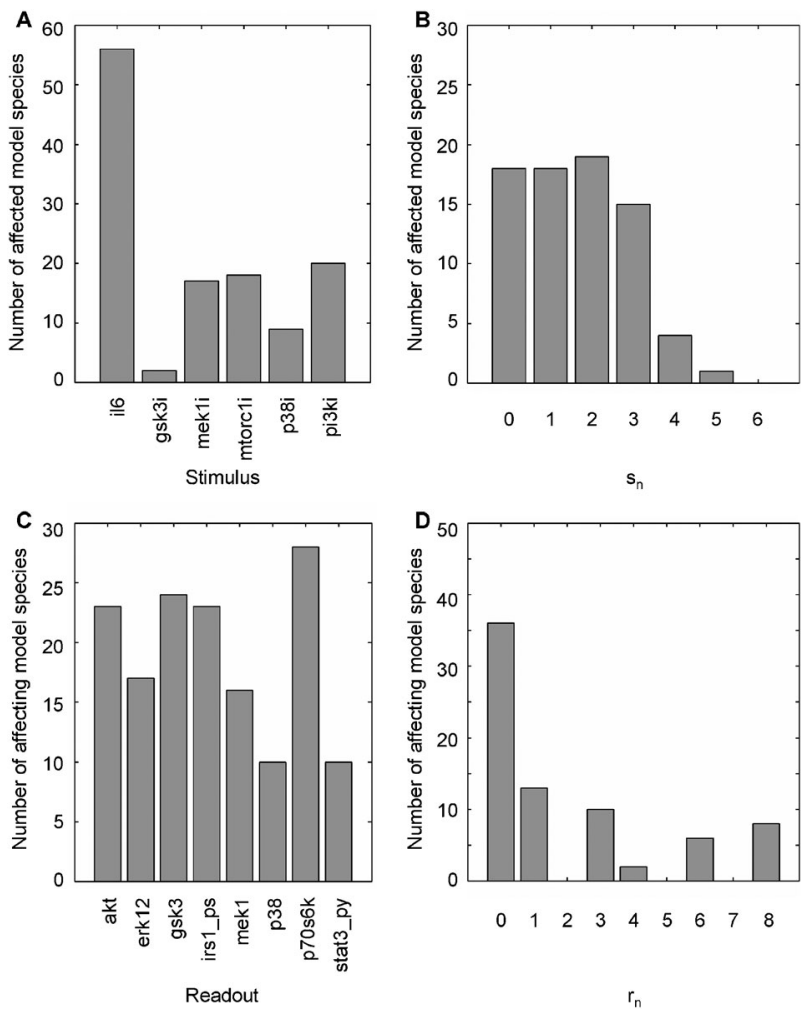

Fig. 11 Experimental coverage of modelled IL-6 receptor signalling. A: Number of network species affected by each of the applied cytokine/inhibitor treatments (stimulus). B: Distribution of numbers of perturbations affecting IL-6 signalling species. C: Number of network species affecting phosphoproteomic readouts. D: Distribution of numbers of readouts affected by species in the IL-6 signalling network. Corresponding dependency matrices are shown in Fig. S15.B and S16.B (ESI $\dagger$ ). (Total number of IL-6-associated species: 75. .) 
even all seven stimuli. As expected, IL- $1 \alpha$ influences the largest set of species ( $>70)$ followed by the inhibitors 'ikkbi' (41) and 'p38i' (30). In contrast, 'gski' affects the state of only three nodes as it is at "the bottom" of the network. Regarding the observability levels $\left(r_{n}\right)$, we see a reduced coverage compared to the perturbations. For 39 nodes no path connecting them with any of the readouts exists. These species include in particular transcription factors or "event nodes" (such as proliferation or degradation of certain proteins) whose activity cannot be reflected by the readouts (see Fig. 1). On the other hand, 7 nodes influence all 9 readouts and are thus well reflected in the set of measurements. Concerning the readouts one sees that 'akt' and 'gsk3' are influenced by the smallest set of nodes (13 and 14, respectively) whereas the measurements of 'irs1_ps' (37), 'ikba' (35), and 'erk12' (34) are affected by a large number of species.

The IL-6 network with its 75 nodes was perturbed by one cytokine and five inhibitors implying a maximal value of 6 for $s_{n}$ whereas the maximum for $r_{n}$ (number of readouts) is 8 . Coverage analysis results with respect to early signalling events are similar to those for IL-1 (see Fig. 11 and Fig. S15.B and S16.B, ESI $\uparrow$ ). Except for the input nodes, all species are directly or indirectly perturbed at least by the cytokine IL-6 and more than $50 \%$ of all nodes can be additionally affected by one or more inhibitors. 'pi3ki' (20), 'mtorci' (19) and 'mek1i' (18) affect the largest number of nodes. Regarding the observability coverage we see again that a considerable number of (mainly output) species does not influence the readouts. Their state can therefore not be captured by the measurements. As 8 nodes affect all readouts, their state is well reflected by the data. Measurements focusing on p70S6K and GSK3 therefore appear to be most efficient for achieving a high coverage of the IL-6 signalling network as they are influenced by 28 and 24 nodes, respectively.

Generally, this type of coverage analysis seems useful to examine the extent to which applied perturbation experiments together with the available readouts are representative (i) for the behaviour of the entire network and (ii) with respect to certain species. In addition, it helps to identify most effective perturbations and readouts.

\section{Conclusion}

By assembling current scientific knowledge on IL-1 and IL-6 signalling derived from various cell types, we reconstructed two network models summarising IL-1- and IL-6-induced signalling cascades. To the best of our knowledge, this has not been conducted before at this scale. The logical approach used for model set-up enabled us to convert simple relation schemes to executable models. Though limited in reflecting kinetic aspects, those Boolean representations are capable of providing general and qualitative insights into global aspects of signal propagation avoiding the need for detailed information on kinetic mechanisms and parameters usually required for quantitative (e.g. ODE-based) modelling. Topological analysis revealed non-intuitive species interdependencies and feedback structures e.g. demonstrating SHP2 and MKP1 to be substantially involved in both negative and positive feedback loops. Qualitative I/O simulations revealing the initial cell response upon ligand binding as well as the computation of failure modes highlighted potential insulin-desensitising effects and positive-regulatory contributions to HGF expression of both interleukins. This corroborates pathophysiological links to insulin resistance and tumourigenesis in case of chronic inflammation. Additionally, negative-regulatory mechanisms, relevant for systemic "calming down" and stabilisation, seem to trigger IL-1-induced, though initially blocked de novo IL-1 release due to suppression of inhibitory kinase activities (e.g. inhibition of ERK by MKP1). Hence, negative feedback loops impede autocrine effects but enable paracrine, intercellular signal propagation indispensable for inflammatory effector cell recruitment. Apart from that and as being a matter of conjecture, visualised IL-1/IL-6 crosstalk-strategies (e.g. MK2regulated IL-6 receptor turnover or SOCS-mediated inhibition of positive-regulatory TRAF6 ubiquitination) indicate mutual and probably post-initial mechanisms to attenuate and thus control the action of the respective inflammatory "fellow player". The presented models fitted phosphoproteomic data describing the initial response of primary human hepatocytes ${ }^{32}$ on interleukin treatment relatively well. Nevertheless, we could also reveal some effects apparently specific to hepatocytes, such as insignificant p38 MAPK activation but a markedly involvement of the Gabl-mediated positive feedback loop studied by Eulenfeld and Schaper ${ }^{56}$ during initial response to IL-6 to name just a few. Moreover, subsequent hepatocellular model specification suggested the integration of ERK-mediated p90RSK activation and associated influences on mTORCl and p70S6K activity similarly induced by both ligands. In addition, model verification pointed to hitherto undescribed IL-1-induced positive regulation of $\mathrm{p} 38$ MAPK by Akt.

Taking the two structurally validated network models of IL-1 and IL-6 receptor signalling in hepatocytes presented here as a basis, we plan to merge both networks in terms of crosstalk scenarios and coinciding effectors (e.g. MAPKs, PI3K/Akt) and direct our attention to further model falsification focusing on cyclic, hence internally regulated and post-initial cellular response. In order to do so, the explicit consideration of time and signal strength will be necessary. $O D E f y^{57,58}$ should in this context be mentioned as a convenient approach for transforming a given logical model into a corresponding set of qualitative ODEs. ${ }^{57,58}$ The resulting dynamic model allows for ODE-based simulations though with respect to and thus still reflecting the logical relationships encoded in the Boolean model. Timeresolved data may be used to identify required parameter sets (which are significantly smaller than those for mechanistic ODE models) completing the construction of the dynamic model which then facilitates studies on essential properties of the transient and quantitative behaviour of signalling cascades. Our network models might therefore mark a suitable starting point for set-up and analysis of ODE-based models.

The mentioned model transformation narrows the gap between discrete and dynamic modelling frameworks, but, at the same time, model complexity will rise in turn requiring more detailed, at least semi-quantitative information on reaction kinetics. Accordingly, detection of functionally coupled species, forming so called equivalence classes introduced by Samaga et al..$^{30}$ and delineating regulatory motifs should be considered for adequate model reduction. Keeping time-consuming 
literature researches associated to manual model set-up in mind, one should also contemplate software approaches automating Boolean model reconstruction and optimisation (e.g. CellNetOptimizer ${ }^{59}$ ) via direct database access and incorporation of high-throughput data.

One important step in our future work is the integration of the IL-1 and IL-6 network models. Herein we focused on highlighting the mutual up- and down-regulation of IL-1 and IL-6 signalling owing to transcriptional regulation and regulatory kinase/phosphatase onset but did not yet consider direct crosstalks. In principle, one could easily merge the two network models and tag common nodes. However, difficulties arise when formulating the Boolean functions for common nodes where IL-1- and IL-6-induced pathways converge. This type of information is rarely available severely hampering the construction of such an integrated logical model. Only the combined experimental perturbation of the IL- 1 and the IL- 6 network would allow for identification of respective logical relationships.

\section{Methods}

\section{Model set-up}

Extensive literature and database (NCBI/PubMed, UniProtKB/ Swiss-Prot, PhosphoSitePlus) research provided the "status quo" regarding IL-1 and IL-6 signalling, irrespectively of a certain cell type, ensuring a biological starting point for setting up the logical master models. In this context, especially the comprehensive IL-1 pathway map developed by Kracht et al. ${ }^{27,28}$ served as a helpful overview.

Well-suited for formalisation, visualisation, and subsequent qualitative analysis of signal transduction networks, usually structured into input (encompassing e.g. cytokines, growth factors), intermediate (e.g. kinases, phosphatases, scaffold proteins) and output layer (e.g. transcription factors, gene products), the Boolean networks studied within this work are represented as logical interaction hypergraphs (LIHs) previously introduced and utilised by Klamt et al ${ }^{30,31,37}$ Accordingly, each signalling species is denoted by a logical, herein solely binary, state variable defining its discrete level of activation $(0 /$ off $=$ inactive or absent vs. $1 /$ on $=$ active or present). Associated interspecific logical relationships are encoded by Boolean functions exclusively using the logical operators AND (.), OR (+), and NOT (!) and complying with the sum-of-products (SOP) notation. ${ }^{31,60}$ Within the models, each interaction/activation event is expressed by means of AND-connected (potentially negated) nodes (= species) triggering the event (activation of a species) to be described. Hence, having several start nodes, they represent hyperarcs in LIH representation. We keep on referring to hyperarcs (or reactions/interactions) even if a reaction's set of start nodes comprises only one species (equivalent to a simple arc in graphs). For effectors that might be influenced/activated by various distinct events, i.e. by several hyperarcs (AND terms), the latter are OR-connected and individually visualised by multiple hyperarcs pointing into the corresponding node. Considering the scope of our model, certain signalling molecules serve as inputs or side effectors, not being regulated by any network species. They are therefore predefined by default values, reflecting basal activities, endogenous occurrences, catalytic indispensabilities, and inhibitory functions. A special node type called reservoir serves to link post-translationally modified proteins (e.g. ubiquitinated scaffold proteins) to a common pool, enabling us to functionally affect all subtypes by simply influencing the reservoir.

Examples supposed to clarify the LIH formalism as well as its visualisation in Fig. 1 and 2 are listed in Table 1.

Occasionally, it is convenient to introduce accessory "dummy species", denoted by triangles in Fig. 1 and 2, to express a logical relationship more efficiently ( $c f$. line 6 in Table 1).

In cases where an interaction could neither be fully approximated by using an AND nor an OR expression due to insufficient or inconsistent biological information we applied an incomplete truth table (ITT) operator ${ }^{31}$ pointing at the "logical overlap" of AND and OR operations (cf. line 7 in Table 1). Affected species are activated/inactivated (1/0), if and only if all positively influencing effectors are active/inactive (1/0), whereas all negated input species are inactive/active $(0 / 1)$. For all other cases, the species' state remains undefined.

\section{Relevance and confidence level}

Moreover, interactions are individually characterised by a relevance level $\tau$, enabling the discrimination between primary $(\tau=1)$ and secondary $(\tau=2)$ events (hence marking an interaction to be relevant for the initial network response or not). Primary events are considered to be active/available during the initial cell response including gene expression, whereas secondary events cover interactions (i) post-initially closing feedback loops, (ii) initiating negative-regulatory events that require the prior onset of species to be inhibited, or (iii) being less relevant for the activation level of the target species or outlining influences of catalytically aberrant enzyme isoforms, respectively.

Removing secondary interactions with relevance level " $\tau=2$ " prior to computations enables us to focus on primary events during simulations. Where required, the decoupling of secondary " $\tau=2$ " events from preceding AND gates $(\tau=1)$ is realised by introduction of "timescale dummy species". These additional nodes are not explicitly displayed in LIH representation but italicised in corresponding SOP notation ( $c f$. line 8 in Table 1).

According to Samaga et al..$^{30}$ nodes within a feedback loop having the shortest distance to the input layer are regarded as associated initialisation points $(z)$. Therefore, interactions closing the cycle in node $z$ are assigned $\tau=2$.

Interactions are furthermore labelled (Table 2) by a confidence level $c$, indicating the extent to which quoted citations underpin the respective effect in hepatocytes (cf. Fig. 1 and 2 and Tables S3.2 and S4.2 (ESI $\dagger$ ) of model documentations).

Complex AND gates were subjectively estimated with respect to the individual confidence levels of interactions involved.

Model implementation was performed with ProMoT, ${ }^{33-35}$ providing the framework for model construction and visualisation subsequently exportable to CellNetAnalyzer $(C N A)^{36,37}$ for model analysis as explained below. Generated results were in turn re-imported to and graphically represented in ProMoT. Mentioned software tools are freely available (for academic use) on the following web sites:

ProMoT: http://www.mpi-magdeburg.mpg.de/projects/promot/ CNA: http://www.mpi-magdeburg.mpg.de/projects/cna/cna.html 
Table 1 Examples illustrating the logical integration of biological relations and constraints

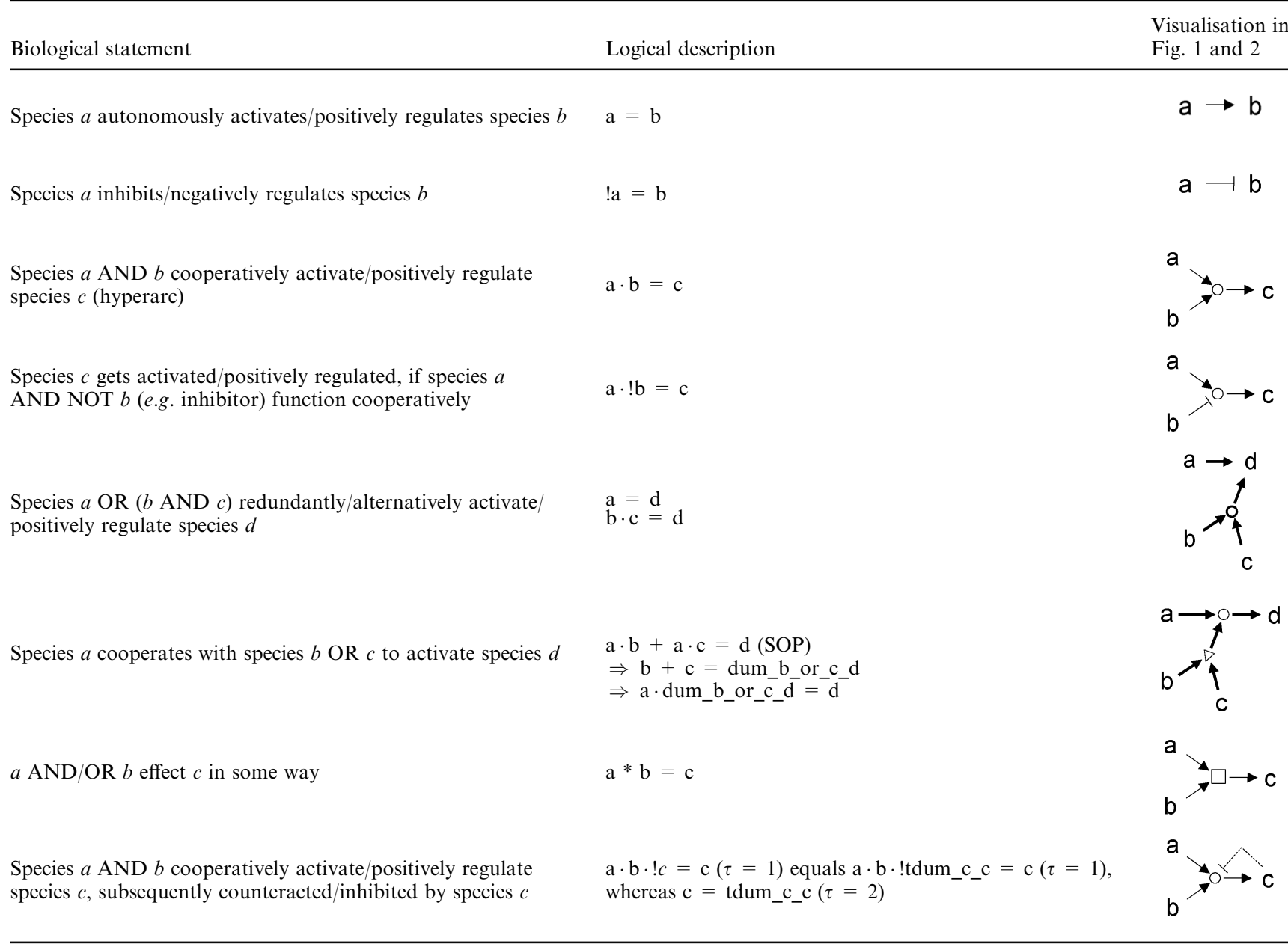

Table 2 Confidence levels and assignment criteria

\begin{tabular}{lll}
\hline & $\begin{array}{l}\text { Primary human hepatocytes, } \\
\text { human hepatoma cell lines }\end{array}$ & Other cell types \\
\hline Stimulus & & \\
IL-1/IL-6 & 1.0 & 0.8 \\
Other & 0.6 & 0.4 \\
\hline
\end{tabular}

For detailed descriptions regarding the model configuration (species, interactions, parameters) and related references see supplementary model documentations (Tables S3.1-4.2, ESI $\dagger$ ).

\section{Topological analysis based on interaction graphs}

By splitting all hyperarcs (AND connections) having more than one start node into simple arcs, we deduced the respective (signed and directed) interaction graphs $(I G s)^{31}$ underlying the logical models introduced here. Edges, entering an AND connection in their negated form, are labelled by a negative, otherwise by a positive sign. Arising duplicate arcs of the same sign were removed to prevent redundancies. As shown by Samaga et al., ${ }^{30} \mathrm{IG}$ analysis suits to studying topological network properties, facilitating for instance the characterisation of feedback loops as well as identification of global species interdependencies. The latter can be stored and visualised in dependency matrices $D .^{31}$
Logical network analysis: input-output behaviour and minimal intervention sets

Using the logical models, we simulated the qualitative inputoutput $(\mathrm{I} / \mathrm{O})$ behaviour of the signalling networks in response to cytokine and/or inhibitor stimulation. In practice, input signals are propagated along the logical links (= hyperarcs), which is equivalent to computing the network's logical steady state (LSS) as described previously. ${ }^{30,31}$ Although influencing cellular responsiveness and behaviour at longer time scales, internal feedback loops may generally impede LSS determination as described by Samaga et al. ${ }^{30} \mathrm{We}$ therefore focused on the initial network response and excluded secondary reactions ( $\tau=2$, see above), usually leading to an acyclic network.

Another useful technique applicable to logical models are minimal intervention sets (MIS). ${ }^{31}$ They are composed of suitable species modifications or exogenous interventions (e.g. constitutive activation or inhibition/knock-out) that enforce a predefined cellular response (e.g. gene expression pattern or phenotype) preferably of pharmacological interest. Restrictions regarding default values, relevance levels etc. can but do not have to be taken into account. A detailed description on the algorithm of MIS computation was given elsewhere. ${ }^{61}$ In this work, the MIS approach was applied to search for 
central regulatory mechanisms impeding uncontrolled cytokine expression, cell proliferation, and viability.

\section{Experimental data}

We used high-throughput experimental data on primary human hepatocytes published by Alexopoulos et al., ${ }^{32}$ generated using multiplexed bead-based ELISA type of assays (xMAP Technology by Luminex, Austin/Texas), and checked for passage-to-passage, preparation-to-preparation, and donor-to-donor variability. The open access MATLAB-based software DataRail ${ }^{62,63}$ was utilised for data management and visualisation. In brief and with regard to species depicted by our models, the phosphorylation state of 11 intracellular signalling molecules was assayed at $t=0$ and $30 \mathrm{~min}$ (relative to cytokine addition) upon cell treatment with IL- $1 \alpha$ or IL-6 in combination with either none or one of 7 small molecule kinase inhibitors (note that inhibitors were added $30 \mathrm{~min}$ prior to cytokine application). For further information on experimental procedures, cytokine and inhibitor concentrations, and data processing see Alexopoulos et al. ${ }^{32}$ and Tables S1 and S2 (ESI $\dagger$ ). The selected data sets applied for validating the IL-1 and IL-6 model are shown in Fig. S9.A and S10.A (ESI $\dagger)$.

\section{Data analysis using interaction graphs}

As delineated by Samaga et al., ${ }^{30}$ we systematically compared measured changes of a protein's $(p)$ phosphorylation state $\left(x^{p}\right)$ assayed at $t=30 \mathrm{~min}$ due to differing treatment scenarios (e.g. $\mathrm{S}_{1}=$ ligand $(l) v s . \mathrm{S}_{2}=$ "add nothing" or $\mathrm{S}_{1}=$ ligand $(l)+$ inhibitor $(i)$ vs. $\mathrm{S}_{2}=$ inhibitor $(i)$ ) with respective IG-derived predictions deduced from entries of the corresponding dependency matrix (see "Topological analysis based on interaction graphs"). For this purpose, we focused on the ratio $x^{p}\left(\mathrm{~S}_{1}\right) / x^{p}\left(\mathrm{~S}_{2}\right)$, quantifying the alteration of species phosphorylation caused by the given stimulus scenarios. For example, in case of an increasing signal owing to ligand application $\left(x^{p}\left(\mathrm{~S}_{1}\right)>x^{p}\left(\mathrm{~S}_{2}\right)\right.$, i.e. $x^{p}\left(\mathrm{~S}_{1}\right) /$ $\left.x^{p}\left(\mathrm{~S}_{2}\right)>1\right)$, one would expect the existence of at least one direct (edge) or indirect (path) positive regulatory influence of species $l$ on species $p(l \rightarrow p)$. Assuming structural consistence within the associated IG, the respective entry of the dependency matrix $D_{l, p}$ (row $l$, column $p$ ) should point to an activating or ambivalent influence. On the other hand, an increase (decrease) in species phosphorylation while applying an inhibitor against associated upstream effectors, suggests the presence of at least one negative (positive) influence path from the inhibited protein $(i)$ to $p(i \rightarrow p)$. Thus, inhibitory effects could be treated analogously, considering row $i$ and column $p$ of the dependency matrix. Differences in signal strengths between $S_{1}$ and $S_{2}$ were taken as significant if the larger signal exceeded the smaller one by at least 50 per cent at $t=30 \mathrm{~min}$, meaning:

$$
x^{p}\left(S_{1}\right) / x^{p}\left(S_{2}\right) \geq 1.5\left(\text { signal increase in } \mathrm{S}_{1}\right)
$$

or

$x^{p}\left(S_{1}\right) / x^{p}\left(S_{2}\right) \leq 1 / 1.5$ (signal decrease in $\mathrm{S}_{1}$ ) for $t=30 \mathrm{~min}$.

Effects appearing beneath or close to technical detection limit $\left(x^{p} \leq 500\right)$ were neglected. Moreover, we preassigned "negative states" to signalling species whose activity is negatively regulated by phosphorylation events (such as GSK3) and inverted related dependency matrix elements accordingly. In consequence, influences like the inhibition of a "negative" species $p$ by ligand $l$ $(I-\mid p)$ as initially depicted by $D_{l, p}$ would than be qualified as positive regulatory events with respect to the measured inhibitory phosphorylation.

\section{Data analysis based on logical models}

In strict accordance with experimental conditions, we furthermore compared measured data (activating vs. inhibitory species phosphorylation) with simulated binary model predictions concerning a species' level of activation. The network's I/O behaviour was calculated as described above. Accordingly, default values (see also "Model set-up") and other initial values reflecting the presence/absence of ligands and/or inhibitors as prescribed by the experimental set-up were fixed.

Inconsistencies between data and modelled $\mathrm{I} / \mathrm{O}$ behaviour may point to errors in the network structure. Following Samaga et al., ${ }^{30}$ raw data were discretised to binary values prior to analysis ensuring their comparability with simulated Boolean states. An effector ( $p$ ) was considered "active or on", if its phosphorylation status assayed at $t=30 \mathrm{~min}$ and caused by a certain stimulus scenario $S\left(x_{S}^{p}(t)\right)$ exceeded each of the following thresholds (based on Samaga et al. ${ }^{30}$ with $t_{0}$ referring to $t=0 \mathrm{~min}$ and $\max \left(x^{p}\right)$ denoting the maximum signal level detected for a species $p$ with respect to all stimulus scenarios):

$x_{S}^{p}(t) / x_{S}^{p}\left(t_{0}\right) \geq 1.5=p_{1} \ldots$ relative significance (signal increase by at least $50 \%$ demanded),

$x_{S}^{p}(t) / \max \left(x^{p}\right) \geq 0.15=p_{2} \ldots$ absolute significance (at least $15 \%$ of the maximum level with respect to all measured stimulus scenarios have to be reached), and

$x_{S}^{p}(t) \geq 500=p_{3} .$. lower detection limit.

Otherwise, the species was set to "inactive or off". Concerning negative regulatory phosphorylation events and associated "negative states" ( $c f$. "Data analysis using interaction graphs"), signals were discretised vice versa, saying $x_{S}^{p}(t)=0$ if all conditions specified above are true and $x_{S}^{p}(t)=1$ elsewise. In principle, the parameters defined above are regarded identical for all signalling molecules studied here, proposing an analytical starting point in want of reference values. Discretised data sets are shown in Fig. S9.B and S10.B, ESI. $\dagger$

\section{Abbreviations (in bold) and molecule names}

$\begin{array}{ll}\text { A20 } & \text { zinc finger protein A20 } \\ \text { ABIN2 } & \text { A20-binding inhibitor of NF- } \mathrm{kB} 2 \\ \text { Akt } & \text { protein kinase B (PKB) } \\ \text { AP-1 } & \text { activator protein 1 } \\ \text { APP } & \text { acute-phase protein } \\ \text { C/EBP } & \text { CCAAT/enhancer binding protein } \\ \text { CaMK II/IV } & \mathrm{Ca}^{2+} / \text { calmodulin }(\mathrm{CaM} \text { )-dependent protein } \\ & \text { kinase II/IV } \\ \text { CCL2 } & \text { chemokine (C-C-motif) ligand 2 } \\ \text { CNA } & \text { CellNetAnalyzer } \\ \text { CRP } & \text { C-reactive protein } \\ \text { EGFR/ErbB } & \text { epidermal growth factor receptor } \\ \text { ERK } & \text { extracellular signal-regulated kinase } \\ \text { FL } & \text { feedback loop } \\ \text { Gab1 } & \text { Grb2 (growth-factor-receptor-binding protein 2)- } \\ & \text { associated binder-1 }\end{array}$




\begin{tabular}{|c|c|}
\hline gp130 & glycoprotein 130 \\
\hline GSK3 & glycogen synthase kinase 3 \\
\hline HGF & hepatocyte growth factor \\
\hline $\mathrm{HNF} 4 \alpha$ & hepatocyte nuclear factor $4 \alpha$ \\
\hline Hsp27 & heat shock protein 27 \\
\hline $\mathbf{I} / \mathbf{O}$ & input-output \\
\hline IFN & interferon \\
\hline IG & interaction graph \\
\hline $\mathrm{IKK} \beta$ & I $\kappa \mathrm{B}$ kinase $\beta$ \\
\hline IL-1 & interleukin 1 \\
\hline IL-1Ra & IL-1 receptor antagonist \\
\hline IL-1RII & interleukin 1 receptor (IL-1R), type II \\
\hline IL-6 & interleukin 6 \\
\hline IL-8 & interleukin 8 \\
\hline iNOS & inducible nitric oxide synthase \\
\hline IR & insulin receptor \\
\hline IRAK & IL-1R-associated kinase \\
\hline IRS1 & insulin receptor substrate 1 \\
\hline ITT & incomplete truth table \\
\hline $\mathrm{I} \kappa \mathrm{B} \alpha$ & inhibitor of nuclear factor (NF) $\kappa \mathrm{B} \alpha$ \\
\hline JNK & c-Jun N-terminal kinase \\
\hline LBP & LPS (lipopolysaccharide) binding protein \\
\hline LIH & logical interaction hypergraph \\
\hline LSS & logical steady state \\
\hline МАРК & mitogen-activated protein kinase \\
\hline MEK & mitogen-activated ERK kinase \\
\hline MEKK3 & MAPK/ERK kinase kinase 3 \\
\hline MIS & minimal intervention set \\
\hline MK2 & MAPK-activated protein kinase 2 \\
\hline MKP1 & MAPK phosphatase 1 \\
\hline $\operatorname{mTOR}(\mathrm{C})$ & mammalian target of rapamycin (complex) \\
\hline MYD88 & myeloid differentiation primary response gene 88 \\
\hline NEMO & $\mathrm{NF}-\kappa \mathrm{B}$ essential modulator \\
\hline$N F-\kappa B$ & nuclear factor $\kappa \mathrm{B}$ \\
\hline ODE & ordinary differential equation \\
\hline p38 & p38-mitogen activated protein kinase (MAPK) \\
\hline p70S6K & ribosomal protein S6 kinase, $70 \mathrm{kDa}$ \\
\hline p90RSK & ribosomal protein S6 kinase, $90 \mathrm{kDa}$ \\
\hline PI3K & phosphatidylinositol 3'-kinase \\
\hline $\mathrm{PKC} \delta$ & protein kinase $\mathrm{C} \delta$ \\
\hline RasGAP & $\begin{array}{l}\text { Ras (v-Ha-ras Harvey rat sarcoma viral onco- } \\
\text { gene homolog) GTPase activating protein }\end{array}$ \\
\hline ROS & reactive oxygen species \\
\hline $\mathbf{S}$ & serine \\
\hline SAA & serum amyloid $\mathrm{A}$ \\
\hline SHP2 & $\begin{array}{l}\text { SH2 domain-containing protein tyrosine } \\
\text { phosphatase } 2\end{array}$ \\
\hline sIL-1RAcP & soluble IL-1R accessory protein \\
\hline sIL-1RI/II & soluble IL-1R, type I/II \\
\hline SOCS & suppressor of cytokine signalling \\
\hline SOP & sum of products \\
\hline STAT & signal transducer and activator of transcription \\
\hline $\mathbf{T}$ & threonine \\
\hline TAB & $\begin{array}{l}\text { TGF (transforming growth factor) } \beta \text {-activated } \\
\text { kinase (TAK)-binding protein }\end{array}$ \\
\hline TAK1 & TGF $\beta$-activated kinase 1 \\
\hline TNF & tumour necrosis factor \\
\hline TPL2 & $\begin{array}{l}\text { proto-oncogene serine/threonine protein kinase } \\
\text { encoded by the tumour progression locus } 2(t p l 2)\end{array}$ \\
\hline
\end{tabular}

$\begin{array}{ll}\text { TRAF6 } & \text { TNF receptor-associated factor } 6 \\ \text { uPA } & \text { urokinase-type plasminogen activator } \\ \mathbf{Y} & \text { tyrosine } \\ \alpha_{2} \mathrm{M} & \alpha_{2} \text {-macroglobulin } \\ \gamma \mathrm{FBG} & \gamma \text {-fibrinogen }\end{array}$

\section{Acknowledgements}

This work was supported by the German Federal Ministry of Education and Research ("The Virtual Liver" project and the FORSYS-Centre MaCS) and the Federal State of SaxonyAnhalt (Research Center "Dynamic Systems").

\section{References}

1 S. W. Chensue, P. D. Terebuh, D. G. Remick, W. E. Scales and S. L. Kunkel, Am. J. Pathol., 1991, 138, 395-402.

2 M. A. Collart, D. Belin, J. D. Vassalli, S. de Kossodo and P. Vassalli, J. Exp. Med., 1986, 164, 2113-2118.

3 C. A. Dinarello, FASEB J., 1988, 2, 108-115.

4 T. Kishimoto, Blood, 1989, 74, 1-10.

5 P. A. Knolle, E. Loser, U. Protzer, R. Duchmann, E. Schmitt, K. H. zum Buschenfelde, S. Rose-John and G. Gerken, Clin. Exp. Immunol., 1997, 107, 555-561.

6 U. Albrecht, X. Yang, R. Asselta, V. Keitel, M. L. Tenchini, S. Ludwig, P. C. Heinrich, D. Haussinger, F. Schaper and J. G. Bode, Cell. Signalling, 2007, 19, 1866-1878.

7 K. Hagihara, T. Nishikawa, Y. Sugamata, J. Song, T. Isobe, T. Taga and K. Yoshizaki, Genes Cells, 2005, 10, 1051-1063.

8 T. Hou, S. Ray and A. R. Brasier, J. Biol. Chem., 2007, 282, 37091-37102.

9 C. J. Kirschning, A. Unbehaun, G. Fiedler, W. Hallatschek, N. Lamping, D. Pfeil and R. R. Schumann, Immunobiology, 1997, 198, 124-135.

10 C. Xia, J. K. Cheshire, H. Patel and P. Woo, Int. J. Biochem. Cell Biol., 1997, 29, 1525-1539.

11 E. Hoffmann, A. Thiefes, D. Buhrow, O. Dittrich-Breiholz, H. Schneider, K. Resch and M. Kracht, J. Biol. Chem., 2005, 280, 9706-9718.

12 S. Wolter, A. Doerrie, A. Weber, H. Schneider, E. Hoffmann, J. von der Ohe, L. Bakiri, E. F. Wagner, K. Resch and M. Kracht, Mol. Cell Biol., 2008, 28, 4407-4423.

13 C. Birchmeier, W. Birchmeier, E. Gherardi and G. F. Vande Woude, Nat. Rev. Mol. Cell Biol., 2003, 4, 915-925.

14 L. Trusolino and P. M. Comoglio, Nat. Rev. Cancer, 2002, 2, 289-300.

15 K. Matsumoto and T. Nakamura, Crit. Rev. Oncog., 1992, 3, $27-54$.

16 G. K. Michalopoulos and M. C. DeFrances, Science, 1997, 276, 60-66.

17 G. Cirillo, L. Casalino, D. Vallone, A. Caracciolo, D. De Cesare and P. Verde, Mol. Cell. Biol., 1999, 19, 6240-6252.

18 J. G. Jiang and R. Zarnegar, Mol. Cell. Biol., 1997, 17, 5758-5770.

19 M. Tamura, N. Arakaki, H. Tsubouchi, H. Takada and Y. Daikuhara, J. Biol. Chem., 1993, 268, 8140-8145.

20 S. Ali, N. N. Singh, H. Yildirim and D. P. Ramji, Int. J. Biochem. Cell Biol., 2010, 42, 113-119.

21 D. Cai, M. Yuan, D. F. Frantz, P. A. Melendez, L. Hansen, J. Lee and S. E. Shoelson, Nat. Med., 2005, 11, 183-190.

22 J. F. Tanti and J. Jager, Curr. Opin. Pharmacol., 2009, 9, $753-762$

23 J. H. Kim, J. E. Kim, H. Y. Liu, W. Cao and J. Chen, J. Biol. Chem., 2008, 283, 708-715.

24 J. J. Senn, P. J. Klover, I. A. Nowak, T. A. Zimmers, L. G. Koniaris, R. W. Furlanetto and R. A. Mooney, J. Biol. Chem., 2003, 278, 13740-13746.

25 M. G. Myers, Jr., R. Mendez, P. Shi, J. H. Pierce, R. Rhoads and M. F. White, J. Biol. Chem., 1998, 273, 26908-26914.

26 F. Andreozzi, E. Laratta, C. Procopio, M. L. Hribal, A. Sciacqua, M. Perticone, C. Miele, F. Perticone and G. Sesti, Mol. Cell. Biol., 2007, 27, 2372-2383. 
27 M. Kracht, A. Weber and P. Wasiliew, Interleukin 1 (IL-1) Pathway, http://stke.sciencemag.org/cgi/cm/stkecm;CMP_21286, Accessed 19 January 2011.

28 A. Weber, P. Wasiliew and M. Kracht, Sci. Signal., 2010, $3, \mathrm{~cm} 1$.

29 J. Saez-Rodriguez, L. Simeoni, J. A. Lindquist, R. Hemenway, U. Bommhardt, B. Arndt, U. U. Haus, R. Weismantel, E. D. Gilles, S. Klamt and B. Schraven, PLoS Comput. Biol., 2007, 3, e163.

30 R. Samaga, J. Saez-Rodriguez, L. G. Alexopoulos, P. K. Sorger and S. Klamt, PLoS Comput. Biol., 2009, 5, e1000438.

31 S. Klamt, J. Saez-Rodriguez, J. A. Lindquist, L. Simeoni and E. D. Gilles, BMC Bioinf., 2006, 7, 56.

32 L. G. Alexopoulos, J. Saez-Rodriguez, B. D. Cosgrove, D. A. Lauffenburger and P. K. Sorger, Mol. Cell. Proteomics, 2010, 9, 1849-1865.

33 ProMoT: http://www.mpi-magdeburg.mpg.de/projects/promot/. Version 0.8.3.

34 S. Mirschel, K. Steinmetz, M. Rempel, M. Ginkel and E. D. Gilles, Bioinformatics, 2009, 25, 687-689.

35 J. Saez-Rodriguez, S. Mirschel, R. Hemenway, S. Klamt, E. D. Gilles and M. Ginkel, BMC Bioinf., 2006, 7, 506.

36 CellNetAnalyzer (CNA): http://www.mpi-magdeburg.mpg.de/ projects/cna/cna.html. Version 9.4.

37 S. Klamt, J. Saez-Rodriguez and E. D. Gilles, BMC Syst. Biol., 2007, 1, 2.

38 J. Saez-Rodriguez, A. Hammerle-Fickinger, O. Dalal, S. Klamt, E. D. Gilles and C. Conradi, IET Syst. Biol., 2008, 2, 80-93.

39 D. Thieffry, Brief. Bioinf., 2007, 8, 220-225.

40 R. Thomas and M. Kaufman, Chaos, 2001, 11, 170-179.

41 R. Thomas and M. Kaufman, Chaos, 2001, 11, 180-195.

42 S. Klamt and A. von Kamp, BMC Bioinf., 2009, 10, 181.

43 A. Ray and B. K. Ray, Mol. Cell. Biol., 1994, 14, 4324- 4332.

44 J. Yao, T. W. Kim, J. Qin, Z. Jiang, Y. Qian, H. Xiao, Y. Lu, W. Qian, M. F. Gulen, N. Sizemore, J. DiDonato, S. Sato, S. Akira, B. Su and X. Li, J. Biol. Chem., 2007, 282, 6075-6089.

45 A. Carriere, M. Cargnello, L. A. Julien, H. Gao, E. Bonneil, P. Thibault and P. P. Roux, Curr. Biol., 2008, 18, 1269-1277.
46 A. Carriere, Y. Romeo, H. A. Acosta-Jaquez, J. Moreau, E. Bonneil, P. Thibault, D. C. Fingar and P. P. Roux, J. Biol. Chem., 2011, 286, 567-577.

47 Y. Shi, J. H. Hsu, L. Hu, J. Gera and A. Lichtenstein, J. Biol. Chem., 2002, 277, 15712-15720.

48 C. C. Dibble, J. M. Asara and B. D. Manning, Mol. Cell. Biol., 2009, 29, 5657-5670.

49 C. Treins, P. H. Warne, M. A. Magnuson, M. Pende and J. Downward, Oncogene, 2010, 29, 1003-1016.

50 S. R. Choudhari, M. A. Khan, G. Harris, D. Picker, G. S. Jacob, T. Block and K. Shailubhai, Mol. Cancer Ther., 2007, 6, 112-121.

51 Y. Wu, J. Liu, Z. Zhang, H. Huang, J. Shen, S. Zhang, Y. Jiang, L. Luo and Z. Yin, Cell. Signalling, 2009, 21, 143-150.

52 J. Landry, H. Lambert, M. Zhou, J. N. Lavoie, E. Hickey, L. A. Weber and C. W. Anderson, J. Biol. Chem., 1992, 267, 794-803.

53 Q. Ding, W. Xia, J. C. Liu, J. Y. Yang, D. F. Lee, J. Xia, G. Bartholomeusz, Y. Li, Y. Pan, Z. Li, R. C. Bargou, J. Qin, C. C. Lai, F. J. Tsai, C. H. Tsai and M. C. Hung, Mol. Cell, 2005, 19, 159-170.

54 V. Stambolic and J. R. Woodgett, Biochem. J., 1994, 303(Pt 3), 701-704.

55 N. Hay and N. Sonenberg, Genes Dev., 2004, 18, 1926-1945.

56 R. Eulenfeld and F. Schaper, J. Cell Sci., 2009, 122, 55-64.

57 J. Krumsiek, S. Polsterl, D. M. Wittmann and F. J. Theis, $B M C$ Bioinf., 2010, 11, 233.

58 D. M. Wittmann, J. Krumsiek, J. Saez-Rodriguez, D. A. Lauffenburger, S. Klamt and F. J. Theis, BMC Syst. Biol., 2009, 3, 98.

59 J. Saez-Rodriguez, L. G. Alexopoulos, J. Epperlein, R. Samaga, D. A. Lauffenburger, S. Klamt and P. K. Sorger, Mol. Syst. Biol., 2009, 5, 331.

60 E. Mendelson, Schaum's outline of boolean algebra and switching circuits, McGraw-Hill, New York, 1970.

61 R. Samaga, A. Von Kamp and S. Klamt, J. Comput. Biol., 2010, 17, 39-53.

62 J. Saez-Rodriguez, A. Goldsipe, J. Muhlich, L. G. Alexopoulos, B. Millard, D. A. Lauffenburger and P. K. Sorger, Bioinformatics, 2008, 24, 840-847.

63 DataRail: http://code.google.com/p/sbpipeline/wiki/DataRail. Version 1.2. 